\title{
Review
}

Dong Zhao, Zhelin Lin, Wenqi Zhu, Henri J. Lezec, Ting Xu, Amit Agrawal, Cheng Zhang* and Kun Huang*

\section{Recent advances in ultraviolet nanophotonics: from plasmonics and metamaterials to metasurfaces}

https://doi.org/10.1515/nanoph-2021-0083

Received February 28, 2021; accepted April 21, 2021;

published online May 24, 2021

\begin{abstract}
Nanophotonic devices, composed of metals, dielectrics, or semiconductors, enable precise and highspatial-resolution manipulation of electromagnetic waves by leveraging diverse light-matter interaction mechanisms at subwavelength length scales. Their compact size, light weight, versatile functionality and unprecedented performance are rapidly revolutionizing how optical devices and systems are constructed across the infrared, visible, and ultraviolet spectra. Here, we review recent advances and future opportunities of nanophotonic elements operating in the ultraviolet spectral region, which include plasmonic devices, optical metamaterials, and optical metasurfaces. We discuss their working principles, material platforms, fabrication, and characterization
\end{abstract}

Dong Zhao and Zhelin Lin contributed equally to this work.

*Corresponding authors: Cheng Zhang, School of Optical and Electronic Information \& Wuhan National Laboratory for Optoelectronics, Huazhong University of Science and Technology, Wuhan, Hubei 430074, China, E-mail: cheng.zhang@hust.edu.cn; and Kun Huang, Department of Optics and Optical Engineering, University of Science and Technology of China, Hefei, Anhui 230026, China, E-mail: huangk17@ustc.edu.cn

Dong Zhao, Department of Optics and Optical Engineering, University of Science and Technology of China, Hefei, Anhui 230026, China Zhelin Lin, School of Optical and Electronic Information \& Wuhan National Laboratory for Optoelectronics, Huazhong University of Science and Technology, Wuhan, Hubei 430074, China

Wenqi Zhu and Amit Agrawal, Physical Measurement Laboratory, National Institute of Standards and Technology, Gaithersburg, MD 20877, USA; and Maryland NanoCenter, University of Maryland, College Park, MD 20742, USA

Henri J. Lezec, Physical Measurement Laboratory, National Institute of Standards and Technology, Gaithersburg, MD 20877, USA

Ting Xu, National Laboratory of Solid State Microstructures \& College of Engineering and Applied Sciences and Collaborative Innovation Center of Advanced Microstructures, Nanjing University, Nanjing, Jiangsu 210093, China

Ә Open Access. ( 2021 Dong Zhao et al., published by De Gruyter. (cc) BY License. techniques, followed by representative device applications across various interdisciplinary areas such as imaging, sensing and spectroscopy. We conclude this review by elaborating on future opportunities and challenges for ultraviolet nanophotonic devices.

Keywords: metamaterials; metasurfaces; nanophotonics; plasmonics; ultraviolet light.

\section{Introduction}

"Ultraviolet" means "beyond violet", where violet refers to the color of visible spectrum with the highest photon energy. Ultraviolet (UV) radiation was discovered by German physicist Johann Wilhelm Ritter in the year 1801 [1]. Ritter observed that invisible rays beyond the violet end of the visible spectrum had an even stronger interaction with silver chloride-soaked papers than the violet light, and therefore called these rays "deoxidizing rays" to emphasize their chemical reactivity. Nowadays, UV radiation typically refers to electromagnetic waves with free-space wavelengths between 10 and $380 \mathrm{~nm}$, and can be further divided into five subbands (Figure 1a), which are the near-UV range [2] (UV-A; free-space wavelength range: $315 \mathrm{~nm} \leq \lambda_{0} \leq 380 \mathrm{~nm}$; photon energy range: $3.26 \mathrm{eV} \leq E_{0} \leq 3.94 \mathrm{eV}$ ), the mid-UV range (UV-B; $280 \mathrm{~nm} \leq \lambda_{0} \leq 315 \mathrm{~nm} ; 3.94 \mathrm{eV} \leq E_{0} \leq 4.43 \mathrm{eV}$ ), the deep-UV range (longer wavelength portion of UV-C; $190 \mathrm{~nm} \leq \lambda_{0} \leq 280 \mathrm{~nm} ; 4.43 \mathrm{eV} \leq E_{0} \leq 6.53 \mathrm{eV}$ ), the vacuum-UV range (shorter wavelength portion of UV-C; $100 \mathrm{~nm} \leq \lambda_{0} \leq 190 \mathrm{~nm} ; 6.53 \mathrm{eV} \leq E_{0} \leq 12.40 \mathrm{eV}$ ), and the extreme-UV range $\left(10 \mathrm{~nm} \leq \lambda_{0} \leq 100 \mathrm{~nm}\right.$; $\left.12.40 \mathrm{eV} \leq E_{0} \leq 123.98 \mathrm{eV}\right)$. UV radiation can be produced by both natural objects such as sun, or artificial sources such as gas-discharge lamps, lasers (e.g., argon-ion laser, helium-cadmium laser, argon-fluoride excimer laser etc.), light emitting diodes [3-5], nonlinear materials [6-11], plasmas [12], and synchrotron [13]. 

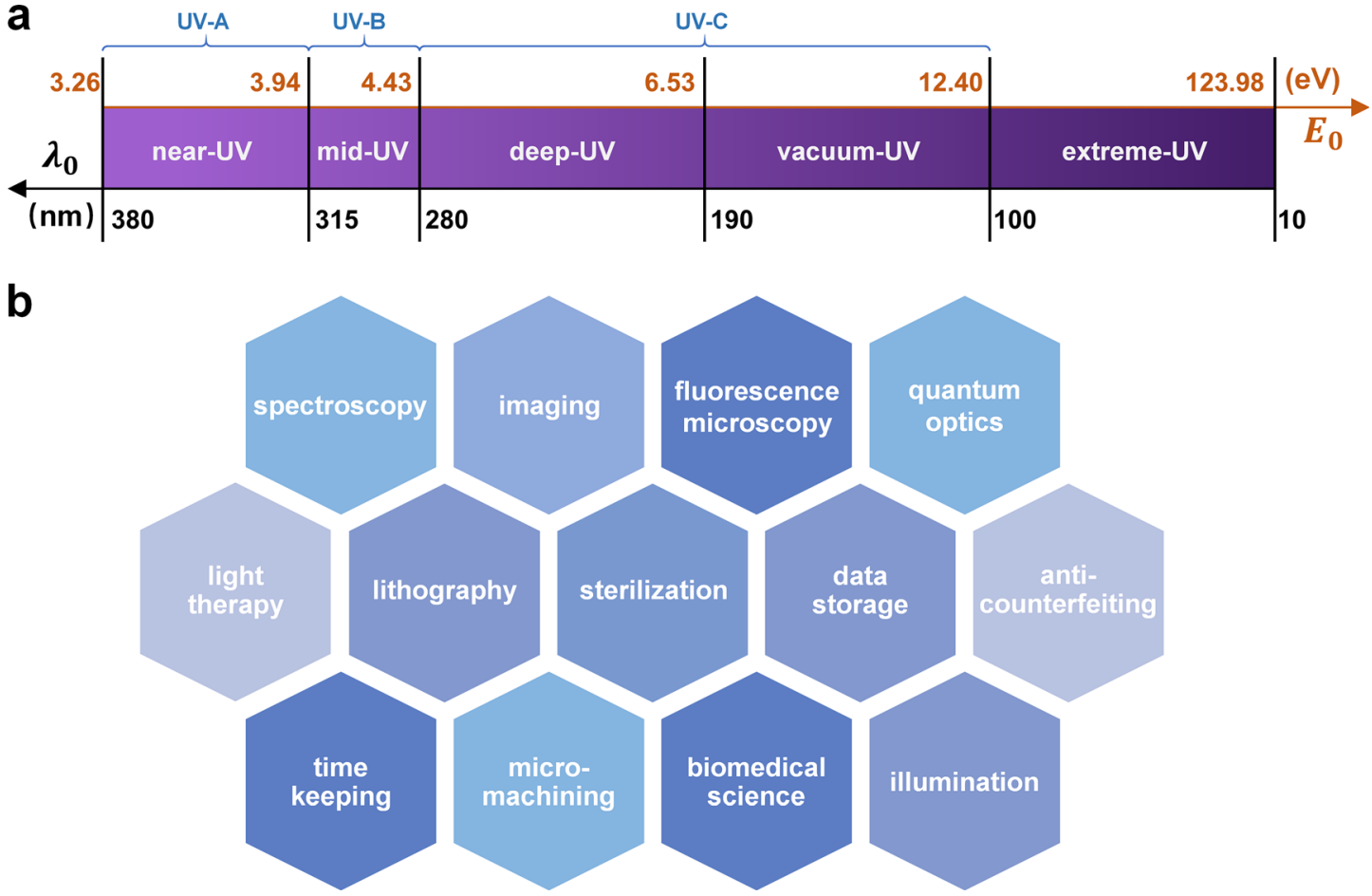

Figure 1: (a) Electromagnetic spectrum for the UV range and the constituent five sub-bands. (b) Representative applications of UV radiation.

UV light plays an irreplaceable role in both fundamental research and practical applications, ranging from spectroscopy, imaging, microscopy, quantum optics, and time keeping, to lithography, light therapy, micromachining, and sterilization (Figure 1b). For example, circular dichroism (CD) spectroscopy using near-UV light is widely used for characterizing the secondary structures of proteins [14], while CD spectroscopy using deep-UV light is largely employed during the exploration and quality evaluation of chiral pharmaceuticals [15, 16]. Similarly, UV spectrophotometry is widely used for nucleic acid quantization [17, 18]. Also, UV light source has long been an essential component in different generations of lithography systems, whose resolution depends critically on the wavelength of the employed UV source [19, 20]. Unlike electromagnetic radiation at lower frequencies, UV radiation can cause ionization and breakage of chemical bonds. Consequently, UV light is widely used for air purification, waste-water treatment, micro-organism, and virus inactivation, etc. [21, 22].

So far, manipulation of UV light largely relies on conventional refractive or reflective optical elements. In contrast to diverse optical materials that are low loss in the near-infrared (near-IR) and visible spectra, suitable materials for the UV region (specially for the deep-UV and even shorter wavelength regions) are relatively limited, expensive, and require complex processing. For instance, high-refractive-index materials are preferred for various optoelectronic applications including antireflection coatings, filters, waveguides, etc. Unfortunately, many high-refractive-index materials are characterized by a relatively narrow bandgap, resulting in a high absorption of UV light. Consequently, UV optical elements are limited in functionality, diversity, operational bandwidth, and manufacturability, in comparison to their near-IR and visible counterparts. While exploring new UV materials and associated manufacturing techniques to overcome the aforementioned limitations, researchers are also exploiting new strategies that are based on nanophotonic technology, including those based on plasmonics, metamaterial, and metasurface concepts. Contrary to conventional optical elements which reflect, refract, or diffract a light beam with their spatially varying shapes, nanophotonic devices modulate various parameters of an electromagnetic wave (e.g., amplitude, phase or polarization state) by engineering their constituent subwavelength unit cells [23-26]. These nanoscale building blocks can be judiciously designed such that the entire device either exhibits an electromagnetic response rarely found in nature, or provides similar or even superior functionality compared to its conventional bulk-optic counterpart, but with a tremendously reduced size. Consequently, 
nanophotonic technology holds great potential in creating high-performance optoelectronic systems with novel functionality, low loss, and compact footprint [27-29].

In this review, we summarize previous achievements of UV nanophotonics technology and discuss its challenges as well as opportunities. We will first elaborate on UV plasmonic devices by explaining their working principle, key characteristics, as well as associated excitation and detection techniques (Section 2). Then, we will review one representative metaoptics element, i.e., metamaterial, and discuss its applications in realizing novel electromagnetic functionalities in the UV, including negative index of refraction and hyperbolic dispersion (Section 3). Afterwards, we show that a UV metasurface can be obtained when a volumetric three-dimensional (3D) UV metamaterial gets condensed to a two-dimensional (2D) layer, and discuss the relevant physical mechanisms, material choices, as well as fabrication methods (Section 4). Different applications of the aforementioned devices, ranging from plasmonics-based photodetection and Raman spectroscopy, to metaoptics-based light-field manipulation (e.g., flat lensing, beam bending, holographic projection), photoemission spectroscopy, and nonlinear signal generation, are surveyed (Section 5). We conclude the review by discussing existing challenges and potential future directions of UV nanophotonics technology.

\section{Ultraviolet plasmonics}

When excited by an external electromagnetic radiation, certain electric conductors such as gold $(\mathrm{Au})$, silver $(\mathrm{Ag})$ and aluminum (Al) support collective oscillations of quasi-free electrons at the metal-dielectric interface [30] (Figure 2a). These oscillations decay exponentially along the normal direction (i.e., $z$ ) away from the interface into both the metal and the surrounding dielectric, but propagates like a quasiparticle bound to the interface (i.e., along $x-y$ plane), thereby being named as surface plasmon polaritons (SPPs). The electric field of an SPP is tightly confined at the interface between the metal and dielectric, which mathematically requires a pure imaginary $z$-component of the wave vector $\mathbf{k}\left(|\mathbf{k}|=2 \pi n / \lambda_{0}\right.$, where $n$ is the complex refractive index of the metal and $\lambda_{0}$ is the freespace wavelength). It means that, for a good metal supporting SPPs, its refractive index $n$ has a dominating imaginary part and an extremely small real part, thus demanding a negative real part in the permittivity. Au and Ag exhibit high-quality SPP resonances in the near-IR and visible regions [31-33], while $\mathrm{Al}$ is suitable for supporting SPP resonances in the UV (whose permittivity curves are shown in Figure 2b) [34]. Other metals such as gallium (Ga), indium (In), tin ( $\mathrm{Sn})$, titanium (Ti), lead $(\mathrm{Pb})$, and bismuth (Bi) have also been explored as alternate UV plasmonic materials as their permittivity also exhibits a negative real part in the UV [35]. Recently, silicon (Si), as a representative semiconductor material, has been found to support SPPs with a photon energy up to $11.4 \mathrm{eV}[36,37]$, which is located in the extreme UV range.

Due to negative real part of the refractive index for plasmonic materials, SPPs have an imaginary in-plane ( $x$ or $y$ ) wave-vector component, and they decay exponentially while propagating along the metal-dielectric interface. According to the band structure of a metal, interband transition of electrons can be induced by incident photons, accompanying energy transfer to kinetic energy of the electrons [39]. During this process, the created hot carriers, electrons, and holes, have lifetimes on the order of several tens of femtoseconds, which is too short to reuse for energy harvesting and detection. In addition, phonon resonance, electron-electron scattering, and Landau damping also contribute to SPP absorption [39]. Although, in principle, UV SPPs can also be excited by using prism coupling, grating coupling, highly focusing optical beam or near-field interaction [30], strong material absorption of the UV light by conventional optics and lowefficiency of photodetectors has hindered development of UV optical systems. So, we constrain our discussion on excitation of UV SPPs to two approaches: using transverse-magnetic (TM)-polarized plane-wave illuminating metals from the dielectric substrate side (Figure 2c), and using accelerated high-energy electron beams (Figure 2d).

The photon approach utilizes TM polarized light to illuminate a thin metal film from the transparent substrate side, at an angle of incidence of $\theta$ (Figure 2c). SPPs can be excited when the momentum conservation relationship between the incident light and SPP is satisfied [30], i.e., $k_{\mathrm{SPP}}=k_{0} n_{d} \sin \theta$, where $k_{0}$ is the free-space wave number, $n_{\mathrm{d}}$ is the refractive index of the transparent substrate, the SPP wavenumber $k_{\mathrm{SPP}}=k_{0}\left[\varepsilon_{r} \varepsilon_{s} /\left(\varepsilon_{r}+\varepsilon_{S}\right)\right]^{1 / 2}$, $\varepsilon_{r}$ is the real part of the refractive index of meal and $\varepsilon_{s}$ is the permittivity of material (e.g., air) above the metal film. In such configuration, SPPs are excited only at the interface between air and metal film via the tunneling effect, which requires that the thickness of the metal film is smaller than the skin depth (usually several tens of nanometers). A nearly perfect coupling from a TM polarized light into the SPPs can be achieved by optimizing the metal film thickness, the angle of incidence, and the illumination wavelength, and will lead to a close-to-zero reflection of the incident TM light. SPPs can be observed by using scanning near-field optical microscopy (SNOM). However, observations of UV SPPs using such 
a
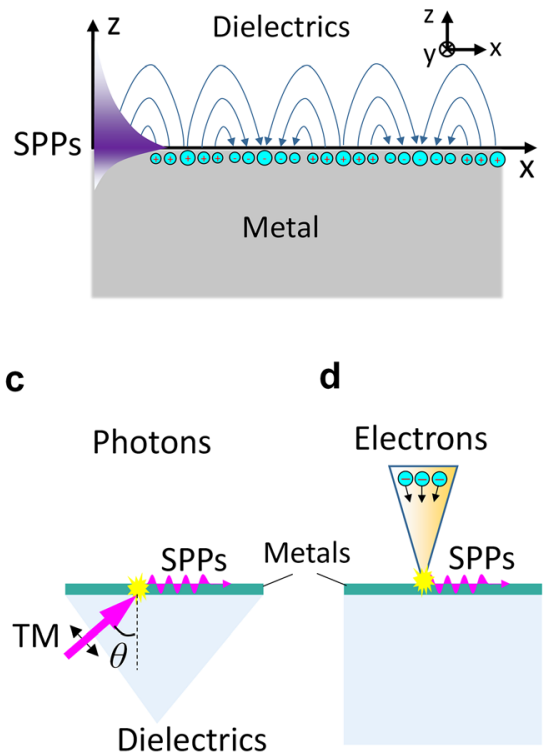

e

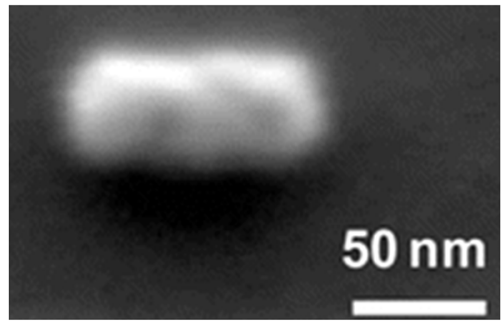

b

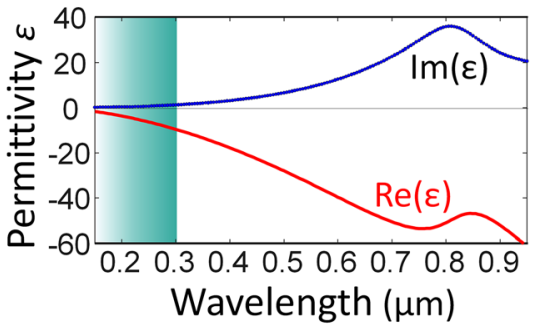

f

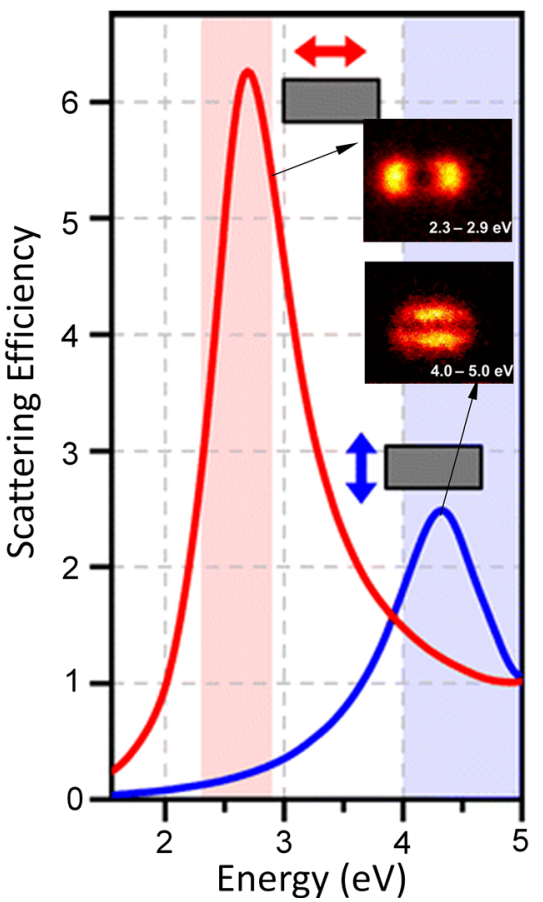

Figure 2: (a) Sketch of SPPs at the interface between dielectric and metal. (b) Permittivity of aluminum. (c and d) Sketch of the excitation of UV SPPs by using photons (c) and electrons (d). (e) SEM image of an aluminum nanorod. (f) The simulated scattering intensity for the longitudinal (red) and transverse (blue) plasmonic modes existing in the aluminum nanorod in (e). Knight et al. [38]. @ 2012 American Chemical Society. The inserts show the experimental measured plasmonics modes with different photon energies.

method have not been reported yet. Instead, Gryczynski et al. employed a 'reverse' process where SPPs are first excited by the fluorescence emission from DNA based analogue 2-aminopurine and then transferred into a freespace propagating waves at an outcoupling angle of $59^{\circ}$, resulting in observation of an optical cone with narrow width at a free-space wavelength of $370 \mathrm{~nm}$ [40].

High-energy electrons incident on metallic nanostructures can also excite SPPs through oscillating free carriers (Figure 2d), and at the same time, create a far-field radiation referred to as cathodoluminescence (CL) [41]. Since the CL intensity depends on the local density of states (LDOS), its measurement enables mapping of plasmon modes with a spatial resolution down to a few nanometers, benefitting from the small focused area of electron beams. Due to its high spatial resolution, electron-beam excitation is considered as the most efficient approach to study plasmonic behavior of individual or multiple nanostructures at UV wavelengths [38]. Moreover, use of high-energy electrons has another advantage of simultaneous excitation and detection of UV plasmon. For example, CL mapping of the LDOS in a metallic nanostructure can reveal both the transverse and longitudinal plasmonic modes through analysis of the CL radiation scattering spectrum [38]. For an Al nanorod shown in Figure 2e, the excited transverse and longitudinal modes exhibit maximum scattering intensity at photon evergies of $\approx 2.6$ and $\approx 4.3 \mathrm{eV}$, respectively (Figure $2 \mathrm{f}$ ).

Finally, it is worth noting that although loss is inevitable for SPPs, the highly confined electric fields at the metal-dielectric interface offer a significant field enhancement, which facilitates observation of weak 
physical effects such as Raman scattering [42], nonlinear process [43] and photocurrent creation [44].

\section{Ultraviolet metamaterials}

Metamaterials typically refer to artificial three-dimensional (3D), volumetric media composed of bulk metallic and/or dielectric constituent elements. Optical metamaterials exhibit electromagnetic responses that are not found in nature and are radically different from those of their constituent materials. For example, a metamaterial acting as a left-handed (LH) or negative-index optical medium can sustain backwards electromagnetic waves, leading to several counter-intuitive phenomena such as negative refraction, reverse Doppler and Vavilov-Cherenkov effects as well as negative radiation pressure [45-47]. A LH medium is characterized by a complex electric permittivity $\epsilon$ and a complex magnetic permeability $\mu$ that are simultaneously negative in their real parts. When an electromagnetic plane wave propagates inside a $\mathrm{LH}$ medium, the direction of power flow (defined by the time-averaged Poynting-vector $\boldsymbol{S}$ ) is opposite to that of phase-front propagation (defined by the wave-vector $\boldsymbol{k}$ ). This yields a "backwards wave" described by a mode index (phase refractive index $n=-\operatorname{Re}[\sqrt{\epsilon \cdot \mu}]$ ) which is negative.

Media with a LH electromagnetic response, though not found to exist in a naturally occurring state yet, are compelling because they entertain the possibility of realizing potentially useful functions such as flat lensing [45] and Veselago-Pendry superlensing [48]. This has triggered a quest to achieve $\mathrm{LH}$ electromagnetic responses via the use of artificial metamaterials, which are usually composed of arrays of lithographically shaped metaldielectric resonators with deep-subwavelength periodicity, and designed for operation at frequencies ranging from the microwave $[47,49]$ up to the red-end of the visible [50]. However, the corresponding negative refractive index is typically narrowband and strongly angledependent, ruling out straightforward use in most potential applications relying on broad-angle off-axis propagation, such as flat lensing and superlensing. Moreover, achieving a resonator-based LH response at higher frequencies of interest for imaging and lithography applications, such as at UV frequencies, is difficult due to fabrication constraints related to miniaturization of resonator dimensions.

An alternative scheme for implementing metamaterials with LH responses is to exploit the bulk-plasmon and surface-plasmon resonances of constituent metal volumes and metal-dielectric interfaces. Using this approach, a metal-dielectric-metal (MDM) plasmonic waveguide was first employed to create a "two-dimensional" LH metamaterial with an isotropic negative index of refraction in the waveguide plane, at green and blue frequencies [51]. The $\omega-k$ dispersion diagram (Figure 3a) of the plasmon mode in an MDM waveguide exhibits a branch of negative slope (corresponding to a negative refractive index) between the bulk plasmon frequency $\left(\omega_{p}\right)$ and the surface plasmon resonance frequency $\left(\omega_{s p}\right)$. The high-frequency cutoff for the LH response occurs at $\omega_{p}$, determined solely by the constituent metal. Therefore, choosing a metal with high $\omega_{p}$ (e.g., Ag, Al) can push the LH response to the UV or deep-UV spectral range. In addition, the low-frequency cutoff occurs at $\omega_{s p}$, which is determined by both the constituent metal and surrounding dielectric, and at the same time, is the frequency at which the real part of the relative permittivity of metal is equal in amplitude but opposite in sign to that of the surrounding dielectric. Therefore, choosing a high-index dielectric such as $\mathrm{TiO}_{2}$ and $\mathrm{Si}_{3} \mathrm{~N}_{4}$ can push the $\mathrm{LH}$ response to the center of the visible spectral range. Silicon (Si), which is another high-index dielectric, can also push the LH response to the red-end of the visible. However, the above-bandgap absorption in Si results in a LH metamaterial with comparably high loss. A clever 2D negative refraction experiment (operating at free-space wavelength $\lambda_{0}=514 \mathrm{~nm}$ ) in an MDM geometry using $\mathrm{Au}-\mathrm{Si}_{3} \mathrm{~N}_{4}-\mathrm{Ag}$ as the constituent materials was performed (Figure 3b) [51]. The clear illustration of negative refraction in this experiment represented the first experimental evidence of negativeindex supported by the plasmon-mode in an MDM waveguide.

It is worthwhile to note that the LH plasmon mode in an MDM waveguide has an antisymmetric mode-profile (Figure 3c), and therefore, can only be excited at nonnormal incidence angles. To circumvent this limitation and to further achieve a bulk, volumetrically quasi-isotropic negative index of refraction in all three dimensions, an alternative approach based on periodically stacked pairs of plasmonic waveguides with an MDMDM configuration was proposed (Figure 4a) [52] and experimentally implemented in the UV for TM-polarized light (Figure 4b) [53]. The experimentally realized 3D metamaterial was designed to exhibit a quasi-isotropic refractive index of -1 at a UV free-space wavelength of $\lambda_{0}=364 \mathrm{~nm}$, and was fabricated by sputtering alternating layers of $\mathrm{Ag}$ and $\mathrm{TiO}_{2}$ onto a fused-silica substrate. The MDMDM geometry can simply be thought of as two coupled MDM stacks, such that the mode becomes symmetric (Figure 4c) and can thus be excited at any angle of incidence both along the plane of the layers and orthogonal to them - making the 
a

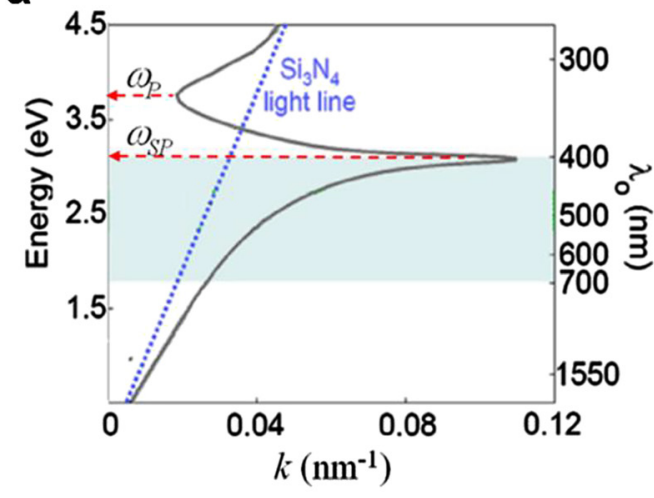

b

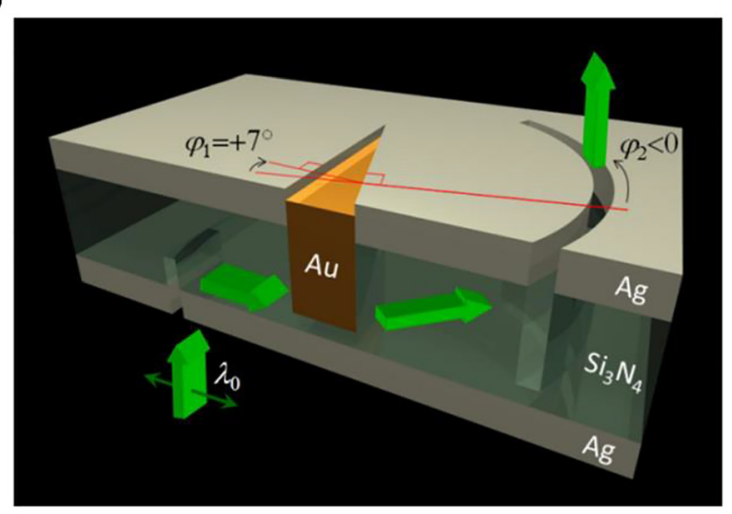

C

\begin{tabular}{|lll|}
\hline Metal & $\mathbf{A g}$ & $\vec{S}$ \\
\hline Dielectric & $\mathrm{Si}_{3} \mathbf{N}_{4}$ & $\stackrel{\vec{k}_{S P P}}{\longrightarrow}$ \\
\hline Metal & $\mathbf{A g}$ & ${ }^{2}$ \\
\hline
\end{tabular}
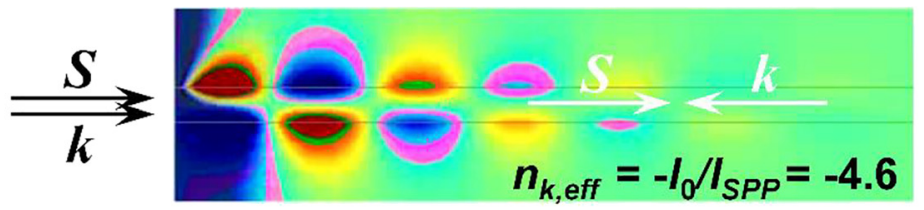

Figure 3: (a) Dispersion diagram for a plasmon mode in an MDM waveguide composed of $\mathrm{Ag}-\mathrm{Si}_{3} \mathrm{~N}_{4}-\mathrm{Ag}$. Lezec et al. [51]. ( $2007 \mathrm{The} A m e r i c a n$ Association for the Advancement of Science. (b) Schematic drawing of negative refraction using Au-prism in an MDM waveguide.

(c) FDTD-calculated magnetic field distribution of the plasmon mode in an MDM waveguide when excited at normal incidence from free-space at $\lambda_{0}=514 \mathrm{~nm}$.

metamaterial behave as a volumetric bulk media with an associated quasi-isotropic index (similar to other isotropic media such as glass or air). In addition to performing negative refraction experiments to determine the refractive index (Figure 4d), researchers further utilized this planar multilayer platform to demonstrate a Veselago flat-lens operating in the UV for the first time (Figure 4e). These experimental realizations of negative refraction and flatlensing using planar multilayer stack of alternating thin films of metal and dielectric represents a paradigm shift in how large-area planar metamaterials can be conceived without relying on complex lithographic patterning requirements.

Remarkably, dispersion engineering with such multilayer approach is not just limited to achieving quasi-isotropic negative index of refraction. Instead, simple tweaking of the layer thicknesses can result in the media acting as a hyperbolic metamaterial (HMM) [54-56]. An HMM is a uniaxial effective medium described by an isotropic relative magnetic permeability $\mu=1$ and diagonal relative electric permittivity tensor that obeys $\epsilon_{\perp}^{\prime} \epsilon_{\|}^{\prime}<0$, where $\epsilon_{\perp}=\epsilon_{\perp}^{\prime}+i \epsilon_{\perp}^{\prime \prime}$ and $\epsilon_{\|}=$ $\epsilon_{\|}^{\prime}+i \epsilon_{\|}^{\prime \prime}$ are respective complex effective relative permittivities for electric field components perpendicular and parallel to the anisotropy axis of the HMM. Depending on which of the two permittivity components, $\epsilon_{\perp}^{\prime}$ or $\epsilon_{\|}^{\prime}$, is negative results in a type I $\left(\epsilon_{\|}^{\prime}<0\right)$ or type II $\left(\epsilon_{\perp}^{\prime}<0\right) \mathrm{HMM}$, either of which can quite elegantly control and/or filter the wavevectors of light propagating inside the HMM. This has led to the use of HMMs in a variety of applications from creating ultrasmall cavities [57], promoting spontaneous emission $[58,59]$ or stimulated emission [60], engineering thermal emission [61, 62], achieving enhanced absorption [63], realizing subdiffraction imaging [64, 65] and facilitating asymmetric transmission [66].

\section{Ultraviolet metasurfaces}

As two-dimensional embodiments of metamaterials, optical metasurfaces are planar nanophotonic devices composed of spatially varying subwavelength nanostructures that could be designed to control the phase, amplitude, wavelength, and polarization of light solely via engineering the geometry and placement of the nanostructures [67-69]. The planarfootprint and subwavelength thickness of metasurfaces enable their fabrication by using one-step top-down lithography technologies used in mature semiconductor industries, which avoids the challenging manufacturing requirements for resonator based three-dimensional metamaterials and therefore pushing metadevices toward practical applications such as optics [70], quantum physics [71-73] and biology [74]. The highly customizable nature of a metasurface allows it to accomplish a variety of functions that have traditionally been fulfilled by a combination of bulk optical elements, such as 
a

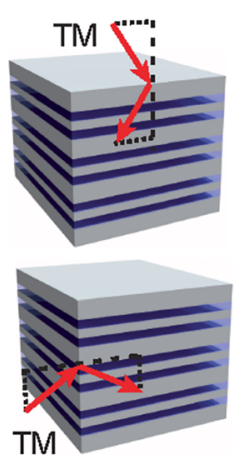

b

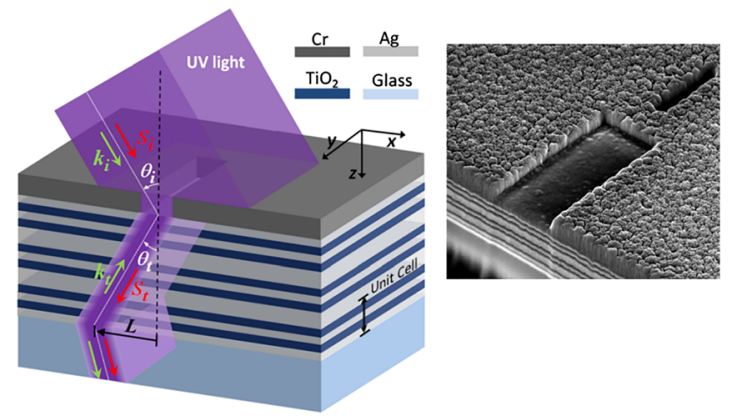

C
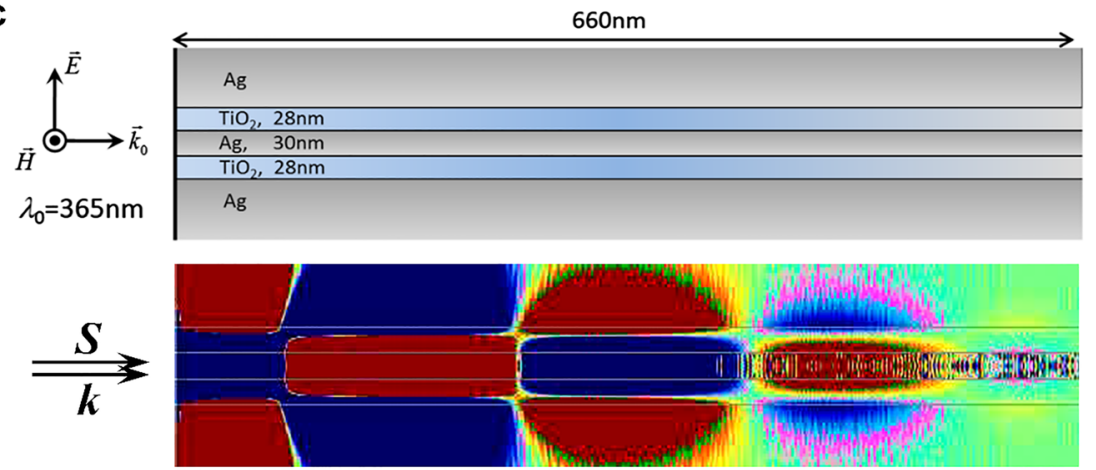

d

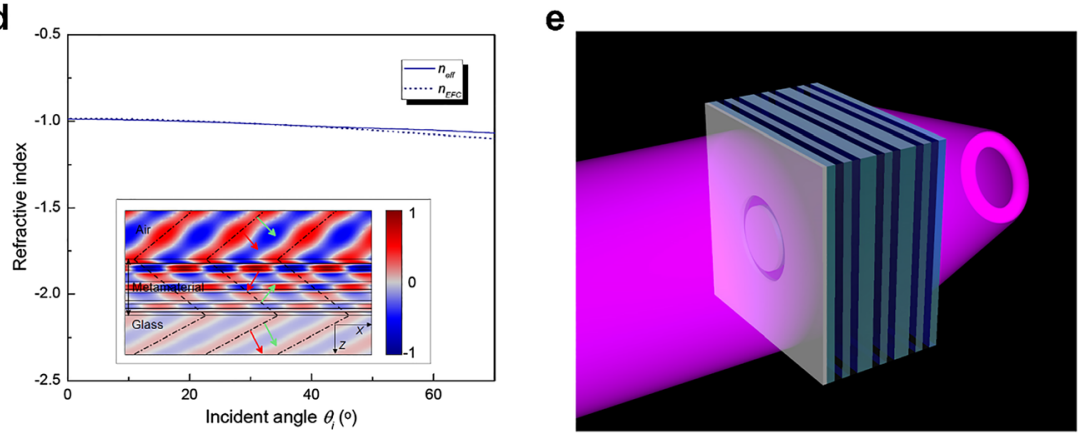

Figure 4: (a) Schematic drawing of all-angle negative refraction for a stack of three MDMDM unit-cells for excitation along the plane of the layers (upper panel), and orthogonal to them (lower panel). TM polarized light is utilized in both cases. Verhagen et al. [52]. () 2010 American Physical Society. (b) Left: schematic drawing of negative refraction from air into a metamaterial formed by three vertically stacked unit cells, each consisting of planar layers of $\mathrm{Ag}-\mathrm{TiO}_{2}-\mathrm{Ag}-\mathrm{TiO}_{2}-\mathrm{Ag}$. The structure is coated with a beam-defining mask consisting of a rectangular aperture (width: $600 \mathrm{~nm}$, length: $10 \mu \mathrm{m}$ ) in an opaque Cr film (thickness: $120 \mathrm{~nm}$ ). Right: scanning electron microscopy image of cross-section of a fabricated device. (c) FDTD-calculated magnetic field distribution of the plasmon mode in an MDMDM waveguide when excited at normal incidence from free-space at $\lambda_{0}=364 \mathrm{~nm}$. (d) Experimental values of refractive index (solid curve), and numerical values of refractive index (dotted curve), as a function of angel of incidence $\theta_{i}$, for TM-polarized light. Inset: FDTD-simulated magnetic field distribution for $\theta_{i}=40^{\circ}$. Xu et al. [53]. () 2013 Springer Nature. (e) Schematic drawing of a flat lens illuminated with UV light (purple). A sample object of ring shape placed on one side of the flat lens is projected as a three-dimensional image in free space on the other side of the lens.

gratings, lenses, polarizers, wave plates, beam splitters, and holograms, with a significantly reduced physical size compared to traditional optical elements. Thus far, researchers have demonstrated various types of highperformance metasurfaces performing an array of functionalities such as beam steering $[67,75]$, high-resolution imaging and display [76-83], hologram projection [84-87], structured light generation [88, 89], asymmetric light transmission [90-92], and temporal pulse shaping [93, 94].

\subsection{Working principles of ultraviolet metasurfaces}

Optical metasurfaces are typically designed according to several different principles, which can be categorized into: (1) propagation phase [95]; (2) geometric phase [87, 96, 97]; or (3) electromagnetic resonances [67, 98]. The principles and applications of metasurfaces operating at visible up to microwave wavelengths have been discussed in several 
previous works [99-104]. Due to high photon energy and strong material absorption of UV light [105], it is relatively challenging to achieve high efficiencies for metasurfaces operating in the UV region. With the advance of nanofabrication techniques and employment of large-bandgap materials, recent years have witnessed an exciting development of compact metasurface-based UV optical elements as well as their diverse applications in holography [106], lithography [107], photoemission spectroscopy [108] and information security [109]. In the following section, we give a brief review of working principles used in the reported UV metasurfaces.

\subsubsection{Waveguide-based ultraviolet metasurfaces}

Waveguide-based metasurfaces usually rely on lowabsorption dielectric nanostructures of high refractive index, which enables the formation of well-defined cavities with low-index background (Figure 5a). Light passing through the dielectric nanostructure propagates in a confined waveguide mode with its propagation properties dependent on the shape and size of the nanostructure. When the lateral size of the planar nanostructure is modified, the corresponding waveguide mode exhibits a different mode volume, mode number, and field profile (Figure 5b), all of which could affect the amplitude and phase of the transmitted light. With the aid of numerical modelling techniques such as finite-difference-timedomain (FDTD) method and finite-element method (FEM), one can obtain the expected amplitude and phase modulation imprinted on the transmitted light for a given waveguide geometry. Since such kind of metasurfaces modulates light via the presence of waveguide modes, we define them as waveguide-based metasurfaces. To realize high operational efficiency in the UV, waveguide-based metasurfaces typically employ large bandgap materials, of which hafnium oxide $\left(\mathrm{HfO}_{2}\right)$ is a representative one. With a bandgap of $\approx 5.7 \mathrm{eV}, \mathrm{HfO}_{2}$ enables a transparency spectral window down to free-space wavelength $\lambda_{0}=217 \mathrm{~nm}[106$, 110]. To achieve both a high optical transmittance and wide range of phase modulation, waveguide-based metasurfaces usually consist of high-aspect-ratio nanopillars [77, 111113]. As an example, the constituent nanopillars in previously-reported $\mathrm{HfO}_{2}$-based waveguide metasurfaces have aspect ratios ranging from 3 to 10 [106]. In many cases, nanopillars of circular in-plane cross-sections are employed for waveguide-based metasurfaces, so that different phase modulation can be implemeted by simply varying the diameter of the pillar. Moreover, thanks to the circular symmetry of its employed pillar, the metasurface's electromagnetic responses exhibit no dependence on the state of polarization of the incident light. Such polarizationinsenstive operation facilitates manipulation of light with various states of polarization, as well as unpolarized light. To realize high performance, the center-to-center spacing between two neighboring nanopillars cannot be too small to minimize coupling between waveguide modes [114]. A rule-of-thumb is to choose the nanopillar peridicity to be approximately one half of the targeted operational wavelength [115].

\subsubsection{Geometric-phase ultraviolet metasurfaces}

Geometric-phase-based metasurfaces are composed of anisotropic nanostructures (Figure 5c) that exhibit different responses to two orthogonal electric-field components (i.e., $E_{x}$ and $E_{y}$ ) of the circularly polarized (CP) incident light [87, 111, 117, 118]. By rotating the nanostructures along a transverse plane, the crosspolarization part of the transmitted or reflected light will immediately harvest a dispersion-free phase that equals twice of the rotation angle. Since this phase is induced by the geometry operation of the in-plane rotation and operates on both components of the cross-polarized light simultaneously, it is usually called as geometric phase or Pancharatnam-Berry phase in memory of their pioneer work [119]. To unveil the underlying physics in geometric phase based metasurfaces, we assume that light passing through individual nanostructures have transmission coefficients of $t_{x}$ and $t_{y}$ for the $E_{x}$ and $E_{y}$ components, respectively. The electric field of CP light has the form of $\mathbf{E}_{\sigma}=E_{0} \cdot[1, \sigma i]^{\mathrm{T}}$, where $\sigma$ denotes the spin of circular polarization, and $\mathrm{T}$ represents the transpose of a matrix. When a nanostructure is rotated by an angle of $\theta$, the vector electric field of transmitted light can be expressed as

$$
\begin{aligned}
\mathbf{E}_{\text {out }} & =E_{0} \cdot \mathbf{R}(-\theta)\left[\begin{array}{cc}
t_{x} & 0 \\
0 & t_{y}
\end{array}\right] \mathbf{R}(\theta)\left[\begin{array}{l}
1 \\
\sigma i
\end{array}\right] \\
& =\frac{t_{x}+t_{y}}{2} \mathbf{E}_{\sigma}+\frac{t_{x}-t_{y}}{2} e^{2 i \sigma \theta} \mathbf{E}_{-\sigma},
\end{aligned}
$$

where the rotation operator $\mathbf{R}(\theta)=\left[\begin{array}{ll}\cos \theta & \sin \theta \\ -\sin \theta & \cos \theta\end{array}\right]$. In eq. (1), one can find that the cross-polarization part in the transmitted light has an additional phase modulation of $2 \sigma \theta$, which is independent of the operating wavelength. It indicates that the geometric phase is the result of the interaction between the spin of photons and the geometric coordinate of the objective physical system. The interaction strength is determined by optical anisotropy of the nanostructures, i.e., $t_{x}$ and $t_{y}$. 

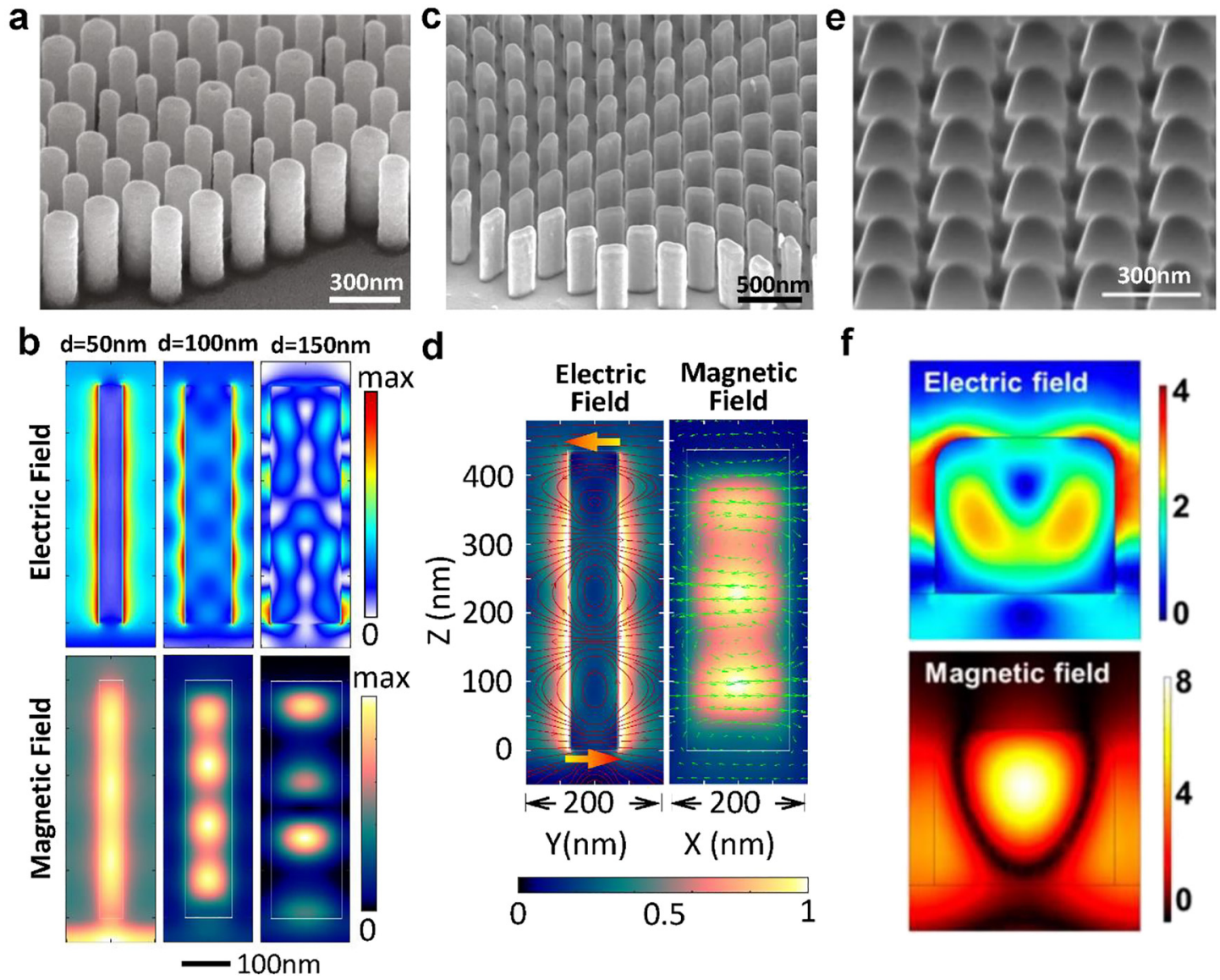

Figure 5: ( $a$ and b) SEM image (a) of $\mathrm{HfO}_{2}$-based waveguide metasurfaces and the electromagnetic nanomodes (b) resonating in the nanopillars with different diameters ranging from $d=50$ to $150 \mathrm{~nm}$. Zhang et al. [106]. Author copyright. (c and d) $\mathrm{SEM}$ image (c) of $\mathrm{Nb}_{2} \mathrm{O}_{5}-\mathrm{based}$ geometric metasurfaces and the antiferromagnetic resonances (d) in the nanostructures under the illumination of $y$-polarized light. Huang et al. [109]. () 2019, WILEY-VCH Verlag GmbH \& Co. KGaA, Weinheim. (e and f) SEM image (e) of the ZnO-based resonance metasurfaces and the simulated magnetic dipoles ( $f$ ) confined in the nanostructure. Semmlinger et al. [116]. (๖ 2018 American Chemical Society.

If $t_{x}=-t_{y}=1$ in eq. (1), the anisotropy is maximized such that the nanostructures work as high-efficiency nanoscale half waveplates due to the phase delay of $\pi$ between two orthogonal electric-field components of light. To enhance the optical transmission of nanostructures, it requires coexistence of both electric and magnetic resonances [120], such that the electric and magnetic dipoles have vertical orientation [98]. In dielectric nanostructures, the magnetic dipoles can be induced by a circulating electric displacement current [121]. It has been found that antiferromagnetic modes containing several antiparallel magnetic dipoles (AMDs) usually appear in nanoscale dielectric half waveplates [109]. More importantly, the AMDs induced by the $E_{x}$ and $E_{y}$ components have odd and even values, respectively. The electric-field vectors of light at the input and output ends of the nanostructure are reversed for odd AMDs (Figure 4d) but stays the same for even AMDs. It implies that the phase delay is $\pi$ for $E_{x}$ but 0 for $E_{y}$, thus realizing the functionality of a half waveplate.
Therefore, such antiferromagnetic resonances hold the fundamental mechanism of nanoscale half waveplates in dielectric geometric metasurfaces. At UV wavelengths, high-efficiency dielectric geometric-phase-based metasurfaces require low-loss materials such as $\mathrm{Nb}_{2} \mathrm{O}_{5}$ [109] or $\mathrm{HfO}_{2}$ [106], which have their transparency windows located at wavelengths $\lambda>340 \mathrm{~nm}$ and $\lambda>217 \mathrm{~nm}$, respectively. At even-smaller wavelengths (vacuum UV and soft X-ray light), all dielectric materials are absorbing [105] hence such antiferromagnetic-resonance-based geometric metasurfaces will not work.

In contrast, the optical anisotropy of a nanostructure is minimum for $t_{x}=t_{y}$ that appears in symmetric, circular-, and square-shaped, nanostructures. In the transmitted light, the cross-polarization component is zero but the copolarization part has no geometric phase modulation. It means that circular and square nanostructures cannot be used for geometric-phase-based metasurfaces. However, if $t_{x} \neq t_{y}$ in eq. (1), the cross-polarization part survives, leading 
to the possibility of demonstrating geometric-phase-based metasurfaces across the entire electromagnetic spectrum. For example, although $\mathrm{Si}$ is highly absorbing at UV wavelengths, a $32 \mathrm{~nm}$ wide and $142 \mathrm{~nm}$ long Si nanorod was demonstrated to work as a UV geometric-phase metasurface element from $\lambda=280 \mathrm{~nm}$ to $\lambda=400 \mathrm{~nm}$ [107], due to the unequal transmission for $E_{x}$ and $E_{y}$ components of light. Even using subwavelength rectangular air-holes etched in a metal film [122], an UV geometric-phase metasurface has been demonstrated without plasmonic resonances at a wavelength of $355 \mathrm{~nm}$. These results suggest that nonresonant geometric-phase-based metasurfaces operating at even shorter wavelengths (such as vacuum UV, deep UV, and soft X-ray wavelengths) are feasible in theory only if the nanostructures have an anisotropy in their shape, i.e., they are of different widths and lengths [122]. Due to high intrinsic absorption of light in materials at such short wavelengths, these nonresonant geometric-phase-based metasurfaces have extremely low efficiencies, e.g., $\approx 0.4 \%$ reported in one previous work [122]. Furthermore, a customized approach to realize phase control of UV light has also been proposed by tailoring the density of noble gas [123]. Note that, the nonresonant geometric-phasebased metasurfaces work under the condition of subwavelength period of unit cells, implying that the feature size of the employed nanostructures must be much smaller than the operating wavelength. The accompanying fabrication issues to experimentally demonstrate a highefficiency UV geometric-phase metasurface is due to the limited patterning resolution of current academic manufacturing technologies. Considering the smallest fabrication feature of several nanometers by using the state-of-the-art industrial equipment and through availability of a low-loss material, the operating wavelength of nonresonant UV geometric-phase metasurfaces can be scaled down to $10 \mathrm{~nm}$, which is located in the extreme-UV spectrum.

\subsubsection{Resonance-based ultraviolet metasurfaces}

Although electromagnetic resonances also exist in waveguide and geometric-phase metasurfaces (Figure $5 b$ and d), higher-order modes containing multiple electric or magnetic dipoles are usually observed in those systems. In fact, a nanostructure supporting single electric or magnetic dipole could also be employed to demonstrate UV metasurfaces. For example, an array of $100 \mathrm{~nm}$ tall and $170 \mathrm{~nm}$ diameter circular $\mathrm{ZnO}$ nanodisks (Figure 5e) is used to induce a magnetic dipole by using circle electric displacement currents at the $395 \mathrm{~nm}$ wavelength [116], as shown in Figure $5 f$.
Similarly, toroidal modes considered as nonradiating monopoles can be excited by metallic meta-atoms composed of two mirror-symmetry ' $D$ '-like nanostructures [43]. Although these two approaches has been demonstrated at UV wavelengths, other methods, such as single electric dipole resonance [124], or simultaneous excitation of electric and magnetic resonances [98] can also be used for UV metasurfaces. From the viewpoint of wavefront shaping, a transmissive metasurface based on only single electric or magnetic dipole can only have a maximum phase modulation of $\pi$ and their efficiency is limited to $<50 \%$ originating from its radiation symmetry [125]. To improve it, both electric and magnetic dipoles oscillating vertically at the transverse plane (perpendicular to the propagation direction of incident light) are needed to exhibit a theoretical maximum transmission of $100 \%$ and a full phase modulation of $2 \pi[98]$. In this case, due to a lack of backward scattering, every meta-atom or nanostructure behaves like a Huygens point source. Therefore, metasurfaces supporting both electric and magnetic dipoles simultaneously are named as Huygens metasurfaces, and have not been reported yet at UV wavelengths. Since these metasurfaces only support one or two electromagnetic modes, we call them as resonancebased metasurfaces to discriminate from the waveguide and geometric-phase-based metasurfaces. Benefiting from small number of modes, resonance-based metasurfaces have small mode volumes, thereby shrinking their thicknesses down to $100 \mathrm{~nm}$ or less, regardless of whether they are based on metallic or dielectric nanostructures.

\subsection{Material platforms and fabrication of ultraviolet metasurfaces}

Silicon (Si), a material which is straightforward to deposit and pattern, and therefore typically used for constructing efficient metasurfaces operating in the infrared (IR) and near-IR region [95, 126], has been employed for building devices operating in the UV [107]. In this study, thin suspended crystalline Si membrane was used to fabricate metasurfaces operating down to the mid-UV and performing beam bending as well as hologram projection. However, device efficiencies were largely limited by significant absorption loss due to the relatively narrow bandgap of $\mathrm{Si}\left(E_{g} \approx 1.1 \mathrm{eV}\right)$. In order to avoid severe interband absorption and realize high-efficiency devices for the UV range, dielectrics of wide bandgap values $\left(E_{g}>3.3 \mathrm{eV}\right)$ are preferred as the constituent materials for metasurface fabrication. Representative wide-bandgap dielectric materials include Silicon Dioxide $\left(\mathrm{SiO}_{2}\right.$, 
$\left.E_{g} \approx 9.0 \mathrm{eV}\right)$, Niobium Pentoxide $\left(\mathrm{Nb}_{2} \mathrm{O}_{5}, E_{g} \approx 3.65 \mathrm{eV}\right)$, Hafnium Oxide $\left(\mathrm{HfO}_{2}, E_{g} \approx 5.7 \mathrm{eV}\right)$, Aluminum Nitride (AlN, $E_{g} \approx 6.0 \mathrm{eV}$ ), and Silicon Nitride $\left(\mathrm{Si}_{3} \mathrm{~N}_{4}, E_{g} \approx 5.0 \mathrm{eV}\right.$ ). The materials' refractive indices in the UV region are plotted in Figure 6a and b. Among them, $\mathrm{SiO}_{2}$ exhibits the lowest refractive index and is readily obtained in the form of high-quality, large-scale wafers. Therefore, $\mathrm{SiO}_{2}$ (usually in the form of fused-silica) is typically employed as the substrate material for UV metasurface devices. In purely numerical studies, AlN and $\mathrm{Si}_{3} \mathrm{~N}_{4}$ have been employed to design metalenses operating down to the deep-UV. In experimental works, $\mathrm{Nb}_{2} \mathrm{O}_{5}$ has been used to construct high-performance devices operating at near-UV wavelengths $\left(\lambda_{0}=355 \mathrm{~nm}\right)$ [109]. Moreover, $\mathrm{HfO}_{2}$, with an even wider bandgap, has been employed to realize efficient metasurface devices operating down to the record-short deep-UV region [106]. Diamond has also been suggested in theory to shape UV light but without any experimental demonstration because of lack of access to large-scale thin diamond film and the associated fabrication challenges, although diamond has a large bandgap of $\approx 5.5 \mathrm{eV}$ and high refractive index of $\approx 2.4$ [127]. Here, we summarize the materials used in UV nanophotonics, as shown in Figure 6e. The small-bandgap materials such as $\mathrm{GaN}$ [78], $\mathrm{TiO}_{2}$ [80] and $\mathrm{Nb}_{2} \mathrm{O}_{5}$ [109] usually work in near-UV region. $\mathrm{Si}_{3} \mathrm{~N}_{4}$ [51], diamond [127], AlN [128], $\mathrm{HfO}_{2}$ [106] work in the deep-UV region due to their bandgaps larger than $4.43 \mathrm{eV}$. In addition, their refractive index is larger than 2, which is sufficient to demonstrate the metasurfaces. In comparison, $\mathrm{CaF}_{2}$ [129], $\mathrm{SiO}_{2}$ [130], and $\mathrm{MgF}_{2}$ [131] with even larger bandgaps have low refractive indices so that they usually are employed as the substrate in UV metasurfaces.

One difficulty of implementing wide-bandgap dielectric-based UV metasurfaces is fabrication of subwavelength-scale, high-aspect-ratio nanostructures. Though directional reactive etching has been demonstrated as a straightforward and effective method to pattern dielectric materials such as $\mathrm{Si}[93,95]$ or $\mathrm{GaN}[78]$, the corresponding etching recipes for patterning many of the aforementioned wide-bandgap materials are instead rather underexploited or even impractical. To overcome such limitations, a resist-based Damascene process has been developed. During this process, metasurface patterns are first created in the resist layer by lithography. Then, low-temperature atomic layer deposition (ALD) is utilized to conformally fill in the holes and trenches of the developed resist layer with the targeted wide-bandgap dielectric material. ALD is intentionally performed over a long duration such that all openings of the exposed resist patterns are completely filled, and at the same time, a quasi-planar dielectric layer is formed on top of the resist layer. Following ALD, the overcoated dielectric layer is back-etched to the resist top surface. Finally, the remaining resist is removed, yielding high-aspect-ratio, straight dielectric nanostructures of uniform height and smooth side-wall profiles (Figure $6 \mathrm{c}$ and d). Utilizing such Damascene process, researchers have successfully implemented $\mathrm{Nb}_{2} \mathrm{O}_{5}$-based devices operating in the near-UV region and performing hologram projection [109], as well as $\mathrm{HfO}_{2}$-based devices operating in the nearand deep-UV regions and performing high-numericalaperture lensing, hologram projection, and Airy beam generation [106].

\section{Applications}

As mentioned above, plasmonic effect as the collective oscillations of electrons is one intrinsic property of material and can be excited with the help of nanostructures. In comparison, metamaterials and metasurfaces are artificial nanostructures that exhibit engineered optical properties of materials. Therefore, the plasmonic materials have been frequently utilized in metamaterials and metasurfaces for various optical devices, where the plasmonic resonances could compensate electromagnetic responses required in metamaterials and metasurfaces. For simplicity of discussion, we only introduce UV devices assisted by the field-enhancement effect of plasmons as well as plasmonic based UV optical elements in Section 5.1, leaving UV plasmonic metamaterials and metasurfaces in Section 5.2. In addition, considering that metamaterials are difficult to fabricate in practical applications, we summarize all applications related to UV metamaterials and metasurfaces in Section 5.2, where metalenses, beam steering, holography, photoemission spectroscopy, and nonlinear optics are addressed with detailed discussions and summary of important advances.

\subsection{Plasmonics-assisted ultraviolet devices}

Highly confined nature of SPPs result in a strong field enhancement at the interface with air in metal films or at the corners or edges of metallic nanostructures. This field enhancement is extremely helpful for various optical effects that depend on the electric field in a nonlinear manner, e.g., facilitating the observation of photon-created carriers in photodetectors, and increasing the optical field 
a

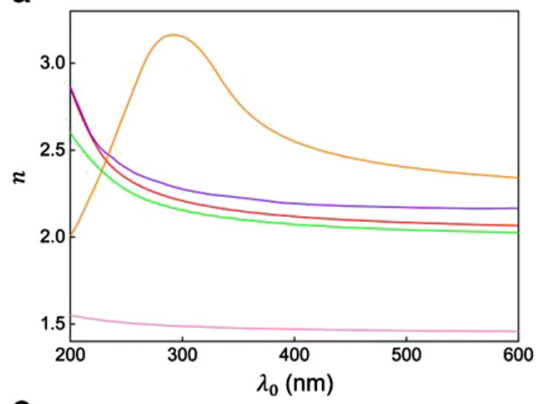

C

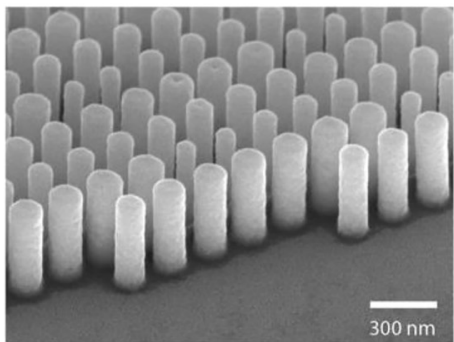

b

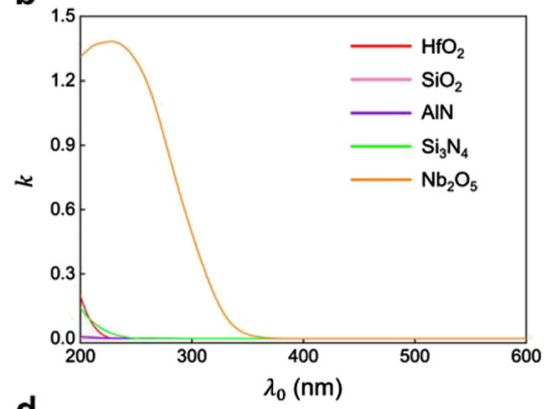

d

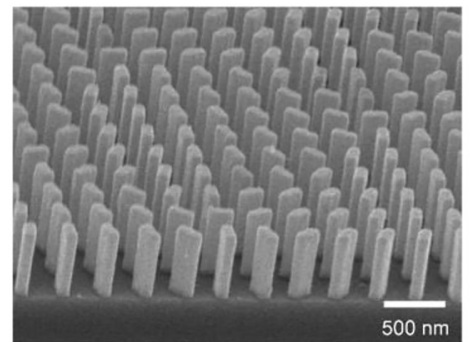

e

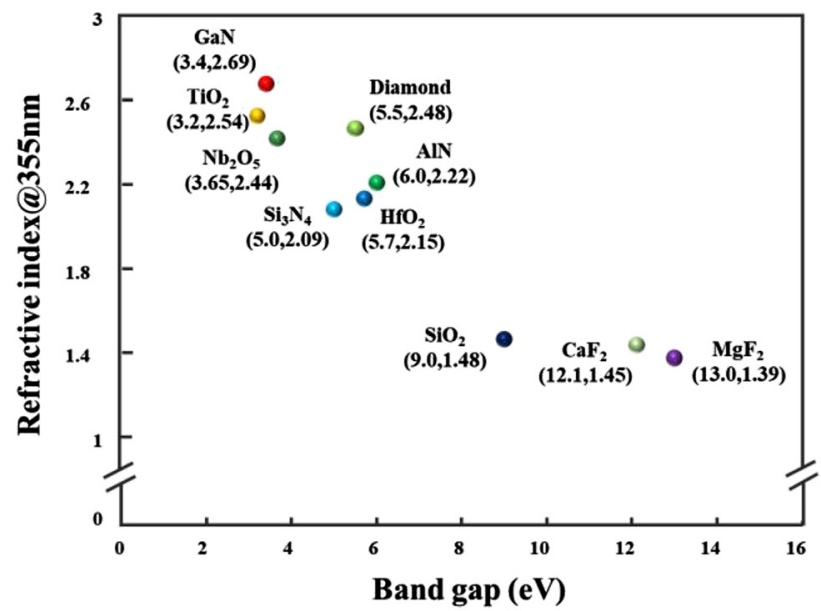

Figure 6: (a and b) Refractive index $n$ (a) and extinction coefficient $k(b)$ of representative UV-transparent dielectric materials. The legend in (b) applies to (a). (c) Scanning electron microscopy image of a $\mathrm{HfO}_{2}$-based metasurface operating in the deep-UV. Zhang et al. [106]. Author copyright. (d) Scanning electron microscopy image of a $\mathrm{Nb}_{2} \mathrm{O}_{5}$-based metasurface operating in the near-UV. Huang et al. [109]. () 2019, WILEYVCH Verlag GmbH \& Co. KGaA, Weinheim. (e) Common optical materials used in UV nanophotonics.

in Raman processes. Here, we introduce the roles of plasmonics in UV photodetectors and Raman spectroscopy.

\subsubsection{Plasmonic ultraviolet photodetectors}

Photodetectors are important optoelectronic devices that transduce incident photons into excited electrons and holes, to create an electric current [132]. The escaped electrons from the surface of materials must overcome its work function, which is relative to the surface potential. Most UV photodetectors adopt a large-bandgap material such as GaN as the host material due to its good chemical and thermal stability enabling a longer lifetime of the created hot carriers [132]. Low dark current and high responsivity are the key properties for photodetectors, but it is difficult to achieve both of them simultaneously [133].
In addition to temperature dependence, low dark current needs large work function of materials to decrease the escaping possibility of the thermally excited electrons. However, the large work function also reduces the escape possibility of the photogenerated carriers, thereby leading to low responsivity. Technically, cooling is the most efficient method to suppress dark current and is typically used in various photodetectors.

In comparison, it is desirable to realize high responsivity UV photodetectors, which will be quite useful for weak-light or single-photon detection for applications in chemistry, quantum optics, and astronomy. In addition to the avalanche-type mechanism, high-responsivity UV photodetectors also employ localized SPPs that provide strong field enhancement [44], which helps to decrease the surface potential of materials around the plasmonic nanoparticles [134]. It decreases the work function and 
increases the escape possibility of photogenerated electrons, therefore enhancing the responsivity. An UV photodetector with gold nanoparticles patterned on the surface of GaN (Figure 7a) exhibit $\approx 30$ times enhancement in the responsivity than the bare GaN-based detector at the UV spectrum ranging from $\lambda=320 \mathrm{~nm}$ to $\lambda=380 \mathrm{~nm}$ (Figure 7b) [44]. Another AlGaN-based UV photodetector with plasmonic effects induced by aluminum nanoparticles has been reported with a twofold enhancement in the responsivity at wavelengths below $300 \mathrm{~nm}$ [135]. More UV photodetectors on the basis of other mechanisms such as avalanche effects and $p-n$ heterojunctions have already been well reviewed in other works $[132,133,136]$.

\subsubsection{Plasmonic ultraviolet surface-enhancement Raman scattering}

Raman scattering is an inelastic light-matter interaction that enables some of the scattered photons to carry slightly different frequencies determined by optical phonons corresponding to the polarizability of molecules or atoms inside solids or some liquids [137]. The scattering intensity at the Raman frequency depends on the polarizability enhancement and the incident intensity. The polarizability of molecules or atoms is an intrinsic property determined by the chemical bonding for a given material and can be seldom modified by incident photons. In comparison, enhancement of the incident intensity is usually achieved by using the plasmonic resonances at rough surface of metals, which is the underlying physical origin of surfaceenhancement Raman scattering (SERS). For a plasmonic surface, the Raman scattering intensity is proportional to the fourth power of the electric-field enhancement factor. Although many SERS spectroscopies have been demonstrated with the silver and gold at the visible and infrared wavelengths, they are not efficient in characterizing the organic and inorganic molecules with small scattering cross section. Meanwhile, the Raman and fluorescence spectra at the visible and infrared can overlay on top of each other, increasing the background noise of Raman signal. Fortunately, SERS signal under UV-light excitation can solve these issues due to its high photon energy.

The first UV SERS was demonstrated with a Raman enhancement of $>2$ orders of magnitude on rhodium and ruthenium electrodes under the $325 \mathrm{~nm}$ wavelength excitation [42]. At the same wavelength, another enhanced SERS was also reported by using Au electrodes [138]. Although these two pioneering works reported exciting

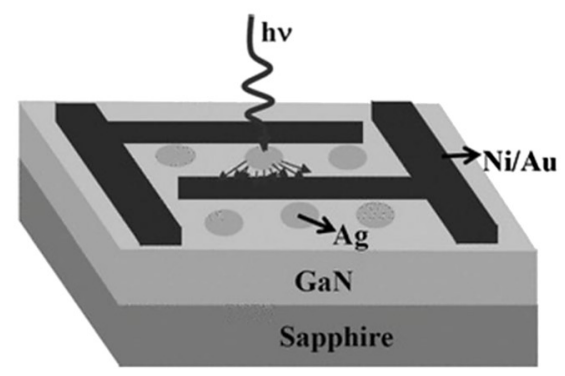

C

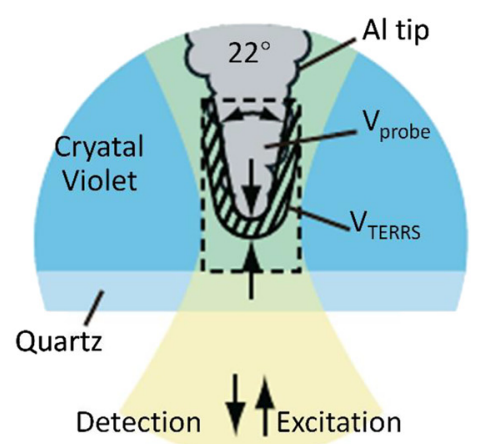

b

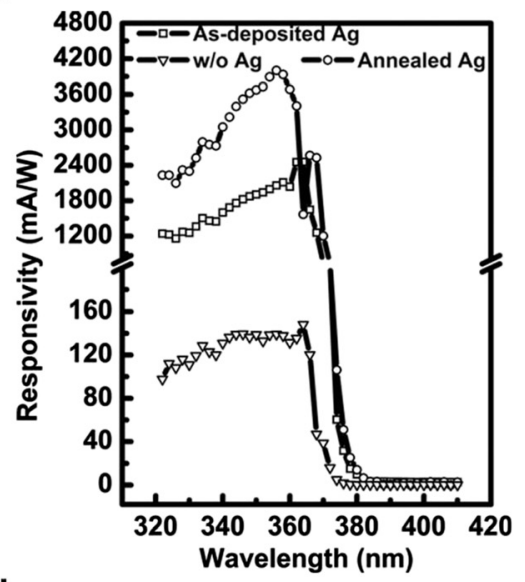

d

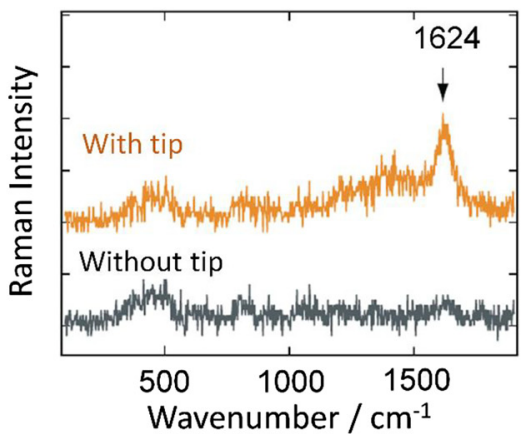

Figure 7: ( $a$ and b) A GaN-based UV photodetector with plasmonic enhancement via Ag nanoparticles (a) and its measured responsivity at the UV wavelengths (b). Li et al. [44]. () 2012 WILEY-VCH Verlag GmbH \& Co. KGaA, Weinheim. (c and d) Tipenhanced Raman scattering spectroscopy (c) and a comparison of the experimental Raman intensity between the cases with and without tip (d). (C) 2009 John Wiley \& Sons, Ltd. 
SERS enhancement results, the role of SPPs during the process was not revealed or discussed comprehensively. Meanwhile, the employed metals such as rhodium, ruthenium, and gold cannot support deep-UV plasmon resonances. A better approach by using a thin $\mathrm{Al}$ layer for the UV SERS was reported in 2007 by Popp's group for an excitation wavelength of $245 \mathrm{~nm}$ [139], which opens more opportunities for UV SERS. For example, an Al-coated nanotip under the $266 \mathrm{~nm}$ deep-UV excitation exhibits a Raman enhancement of $\approx 60$ to 200 times for various samples such as crystal violet solution and films [140], as shown in Figure 7c and d. In addition, benefitting from resonant Raman effects, caused by the fact that the bandgaps of some DNA and proteins are located at the UV spectrum, the Raman signal of adenine molecules had an enhancement of $\approx 5$ on the top of an $\mathrm{Al}$ nanoparticle array [141], compared with the case on the fused silica. Meanwhile, the achieved signal-to-noise ratio of $\approx 50$ indicates that the UV SERS spectroscopy holds the great promise for ultrasensitive detection and characterization of biological issues and molecules.

\subsection{Ultraviolet metaoptics}

Due to the challenge of material absorption and fabrication constraints, the development of UV metasurfaces has only begun since 2018 [43, 107, 116]. Driven by the requirements for industrial applications, UV metasurfaces have harvested increasing attention due to its compact volume and powerful wavefront shaping capabilities. Novel UV metadevices have been demonstrated for various applications such as lenses, beam steering, holography, photoemission spectroscopy and nonlinear phenomena. Here, we review the experimental advances of these UV metadevices.

\subsubsection{Metalens}

Conventional optical lenses control the wavefront of light through a continuous phase gradient that is accumulated when light propagates through a transparent optical medium having spatially varying thickness. This working principle yields bulky three-dimensional (3D) material profiles that are challenging to manufacture within the required wavefront error tolerance, as well as to integrate into compact, miniaturized systems. In contrast, metasurfacebased lenses (metalenses) take a radically different approach to achieve the same functionality and output phase profile, which is by using a planar array of phaseshifting elements of subwavelength dimensions and spacing [142-144].
$\mathrm{HfO}_{2}$ has been employed to construct high numerical aperture $(\mathrm{NA}=0.6)$ metalenses operating at near-UV wavelengths of 364 and $325 \mathrm{~nm}$ (Figure 8a). The nanoscale building blocks of the UV metalenses consist of highaspect-ratio $\mathrm{HfO}_{2}$ circular nanopillars of varying radii arrayed on a fused silica substrate with subwavelength spacing. Each element acts as a truncated dielectric waveguide, through which light propagates with transmission intensity and phase shift controlled by the cylinder height, cylinder radius, and lattice spacing. For the targeted operation wavelength of $364 \mathrm{~nm}(325 \mathrm{~nm})$, a cylinder height of $550 \mathrm{~nm}(500 \mathrm{~nm})$, lattice spacing of $200 \mathrm{~nm}(190 \mathrm{~nm})$, and radius variation range of $50-160 \mathrm{~nm}$ (50 -150 nm) are chosen. For both devices, the measured intensity distribution reveals lensing with a circularly symmetric focal spot, of first-dark-ring diameter close to the theoretical diffraction-limited value (Figure $8 \mathrm{~b}$ and $\mathrm{c}$ ). The focusing efficiency, defined as the ratio of the optical power in the focused spot to the total power illuminating the metalens, are $55.17 \%$ (for the $364-\mathrm{nm}$ metalens) and $56.28 \%$ (for the 364-nm metalens).

\subsubsection{Metasurface-based beam steering}

Traditionally, changing the propagation direction of light can be realized by using optical reflection with a mechanically rotating mirror that is the key element of Galvo scanning systems in optical display projectors, microscopes, and LiDAR. Such a traditional mechanical approach is usually bulky, which is not preferred for portable or integrated systems. Although mirrors based on microelectromechanical systems (MEMS) could shrink the length scale to several tens of micrometers, the tuning angle for most commercial MEMS mirrors is limited to below $\pm 20^{\circ}$, which are insufficient in many applications such as large-area imaging or intelligent vehicles. The alternative method for beam steering is to add an additional tiling phase into the incident beam by using a pure-phase element, such as a spatial light modulator (SLM) [145] or using metasurfaces. The tilting angle is determined by the period of the phase element. The smallest pixel pitch of SLMs is $\approx 3.74 \mu \mathrm{m}$ (Holoeye Gaea), which allows the maximum tilting angle of only a few degrees at the UV and visible wavelengths. Due to the subwavelength pixel pitch, metasurfaces are one of the promising candidates for large-angle steering. Figure 9a and $\mathrm{b}$ sketches Si-based geometric-phase-based metasurfaces composed of nanorods with their orientation angle dependent on the location [107]. Within a period of $160 \mathrm{~nm}$, eight nanorods are used to realize a full-phase modulation of $2 \pi$, resulting in a tilting angle of $\pm 13.1^{\circ}$ at 


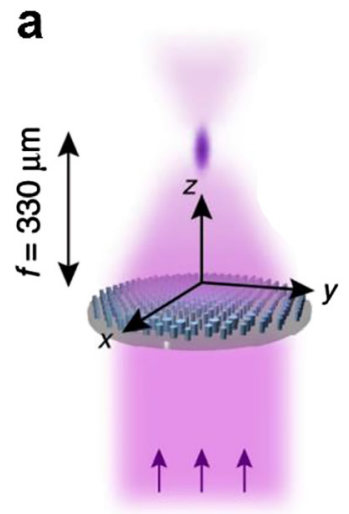

b
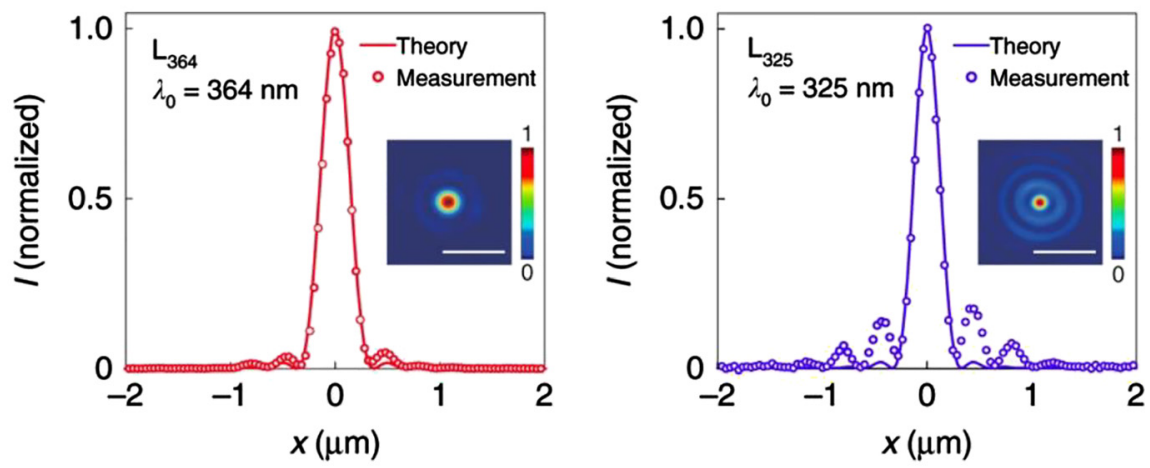

Figure 8: (a) Schematic drawing of focusing by a UV metalens, $L_{364}$ or $L_{325}$, under normal-incidence, plane-wave illumination at free-space wavelength of $\lambda_{0}=364$ or $325 \mathrm{~nm}$, respectively. (b and c) Cross-focus cuts and intensity distributions in the focal plane, as measured for metalenses $L_{364}$ and $L_{325}$, respectively. The theoretically predicted cross-focus cuts are plotted for reference. Scale bars: $1 \mu \mathrm{m}$. Zhang et al. [106]. Author copyright.

$\lambda=290 \mathrm{~nm}$ and $\pm 17.3^{\circ}$ at $\lambda=380 \mathrm{~nm}$ (Figure 9c). Their experimentally demonstrated efficiency is $\approx 10 \%$ from $\lambda=280 \mathrm{~nm}$ to $\lambda=420 \mathrm{~nm}$ (Figure $9 \mathrm{~d}$ ). The tilting angle can be further enlarged by decreasing the number of nanorods in a full phase cycle of $2 \pi$, which, however, decreases the efficiency of the tilting beam. Considering the strong absorption of silicon at the UV spectrum, the upper limit on efficiency is $\approx 10 \%$.

$\mathrm{HfO}_{2}$ with a bandgap of $5.7 \mathrm{eV}$ is a low-loss material platform for the wavefront shaping of light. The waveguide metasurfaces made of circular $\mathrm{HfO}_{2}$ nanopillars have been reported to create an UV Airy beam with a cube phase profile that could make the light beam propagate along a curved trajectory without any significant divergence [106], illustrating a nondiffraction feature (Figure 9e). The measured intensity profiles and the experimental trajectories at various wavelengths are shown in Figure of and g, respectively. Benefiting from the large bandgap, their measured efficiency is $\approx 47 \%$ at $\lambda=364 \mathrm{~nm}$ and $\approx 67 \%$ at $\lambda=325 \mathrm{~nm}$, which are the highest efficiency at these selected wavelengths and therefore makes UV metasurfaces comparable to visible and infrared metasurfaces.

\subsubsection{Metaholography}

As another demonstration of wavefront engineering, UV metasurface holograms have drawn a great deal of attention due to their potential applications in information security and lithography. All reported UV metaholograms employ geometric phase that can be easily controlled by the orientation of a fixed-size nanostructure, which facilitates fabrication due to uniformity in nanostructures shape and size. By using semiconductor
$\mathrm{Nb}_{2} \mathrm{O}_{5}$ with a bandgap of $\approx 3.65 \mathrm{eV}$, an UV metahologram operating at $355 \mathrm{~nm}$ wavelength has been reported with an experimental efficiency of $\approx 79.6 \%$ [109], which is a record-high efficiency for all UV metasurfaces and metadevices demonstrated till date (Figure 10a and b). Another geometric metahologram by using $\mathrm{HfO}_{2}$ nanorods extends the operating spectrum down to $\lambda=325 \mathrm{~nm}$ and $\lambda=266 \mathrm{~nm}$ (Figure 10c and d) [106], meanwhile harvesting impressively high efficiency $(\approx 71.78 \%$ at $\lambda=325 \mathrm{~nm}$ and $\approx 60.67 \%$ at $\lambda=266 \mathrm{~nm}$ ). Both works verify that the UV metasurfaces have a great potential for practical applications because they have solved the efficiency problem. Note that, although both materials $\mathrm{Nb}_{2} \mathrm{O}_{5}$ and $\mathrm{HfO}_{2}$ have large bandgap, their refractive indices are $\approx 2$, which is slightly higher than $\mathrm{SiO}_{2}$ or quartz substrate. It leads to a requirement of high-aspect-ratio nanostructures, e.g., 7 for $\mathrm{Nb}_{2} \mathrm{O}_{5}$ nanobricks [109] and 3-10 for $\mathrm{HfO}_{2}$ nanorods [106], which are challenging to fabricate via the dry etching process. Therefore, both works utilize the aforementioned electron-beam lithography (EBL) combined with ALD technique to fabricate the metasurfaces.

Ultraviolet geometric-phase-based metasurfaces could also realize polarization-multiplexed holograms because the geometric phase is dependent on the handedness of the input circular polarization. A spin-multiplexed metahologram operating at a wavelength of $266 \mathrm{~nm}$ is shown to create two spin-dependent images. When a left-handed CP light illuminates the metahologram, an image of "deep" is constructed at a distance $z=40 \mathrm{~mm}$ away from the metasurface (Fresnel region of the metahologram with a size of $330 \mu \mathrm{m} \times 330 \mu \mathrm{m}$ ), while the right-handed CP light yields the different image of "UV" (Figure 10e) [106]. The phase loaded on the hologram is the weighted super- 
a

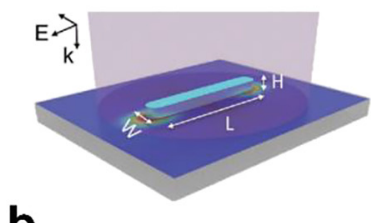

b

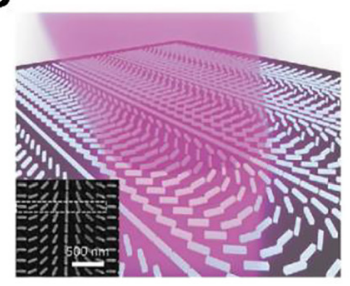

e

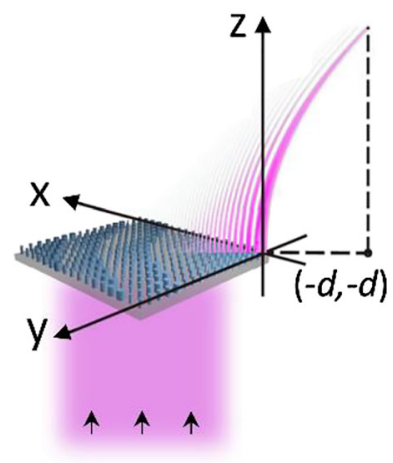

C

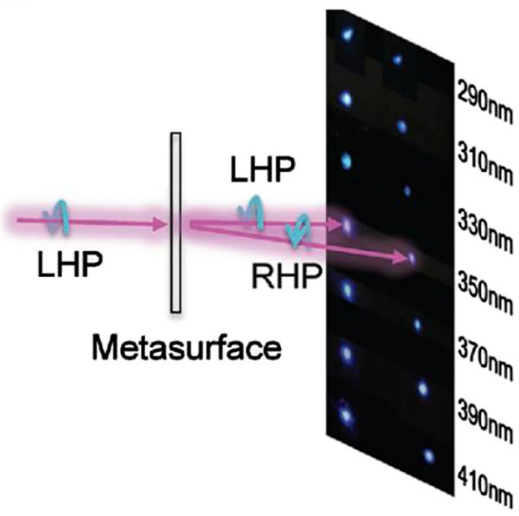

f

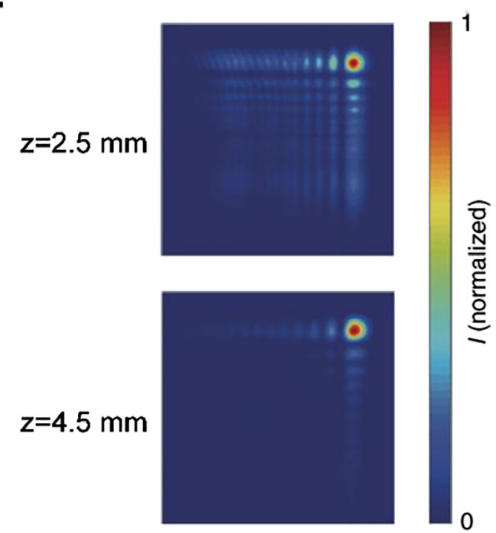

d

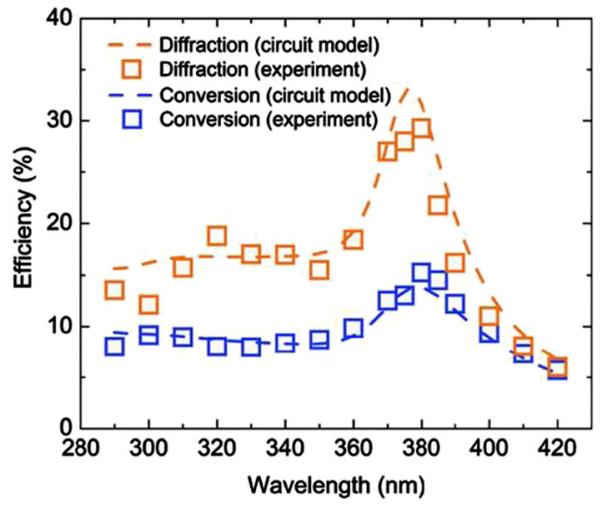

g

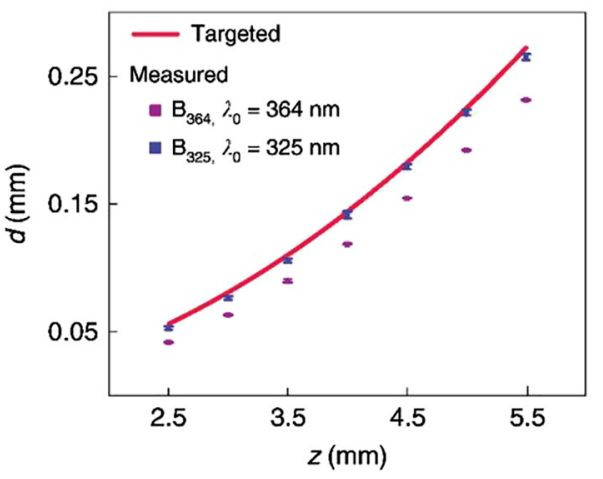

Figure 9: Beam steering by using UV metasurfaces. (a) Sketch for the working principle of Si-based UV geometric metasurfaces. (b) Image of UV metasurfaces for beam steering. (c) Experimental results for steering the UV at the different wavelengths. (d) Measured efficiency of this metasurfaces over a broadband spectrum. Deng et al. [107]. ( 2018 WILEY-VCH Verlag GmbH \& Co. KGaA, Weinheim. (e and f) Sketch for steering the beam along a curved trajectory (e) via the Airy beam (f). (g) A comparison between the experimental and ideal trajectories at the different $z$ cut planes. Zhang et al. [106]. Author copyright.

position of both phase profiles for left- and right-handed CP light, and is used to determine the orientation angle of nanostructures in the metahologram. In comparison, another spin-multiplexed UV metaholograms employs two interleaved apertures [109], each of which encodes two spin-dependent sparse holograms (Figure 10f). One sparse metahologram for left-handedness $\mathrm{CP}$ light reconstructs an image of "girl" pattern at the Fresnel region, while the other hologram for right-handedness $\mathrm{CP}$ light creates the complementary "snake" pattern. Both "girl" and "snake" patterns are complementary to each other so that their combined pattern is observed as another image of "trophy". Thus, one can directly see a picture of "trophy" under the linear-polarization illumination. After the polarization analysis, the pattern "trophy" exhibits a vector feature (Figure 10g), which can provide an additional degree of freedom for the purpose of optical anticounterfeiting. It is attributed to the spin multiplexing functionality of geometric metasurfaces. Note that, both spin-multiplexing approaches realize the holographic reconstruction at the Fresnel region, where the image is real for one spin but becomes virtual for the reversed spin [96, 146]. In contrast, the Fraunhofer metaholograms have center-symmetric real images for both spins [147]. A united mathematical explanation has been given in a newly published book [148].

In addition to optical anticounterfeiting, UV metaholograms has also been used for optical lithography because high energy of UV photons can help to break chemical bonds of molecules in some photoresists such as polymethyl methacrylate (PMMA) and Hydrogen silsesquioxane (HSQ). An ultrathin (40 nm thickness) Si metahologram operating at $\lambda=380 \mathrm{~nm}$ creates the image "Cal", which is used to expose the photoresist [107]. The experimental results are shown in Figure 10h, which indicates good performance. Considering the interference speckles created in the holographic image, a dynamic random phase provided by a rotating optical diffuser is used to increase the coherence of the diffracted light, thus 
a

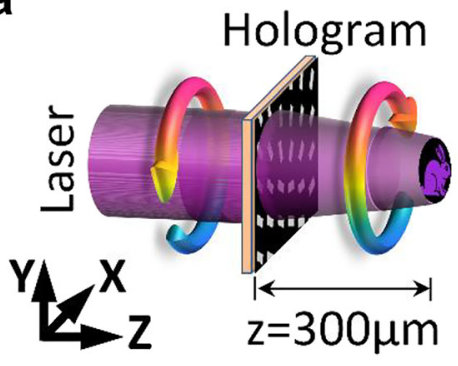

b

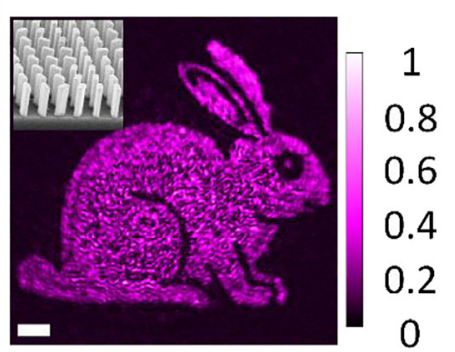

1

6

.4

.2
C

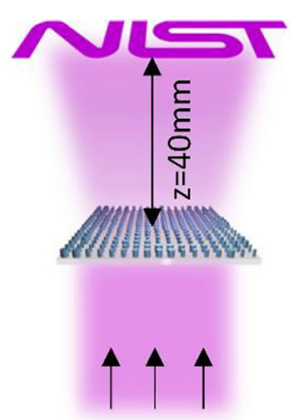

f

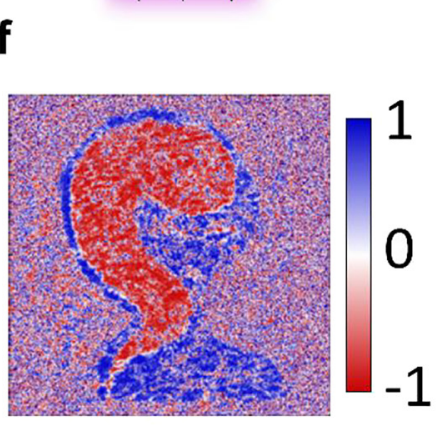

d

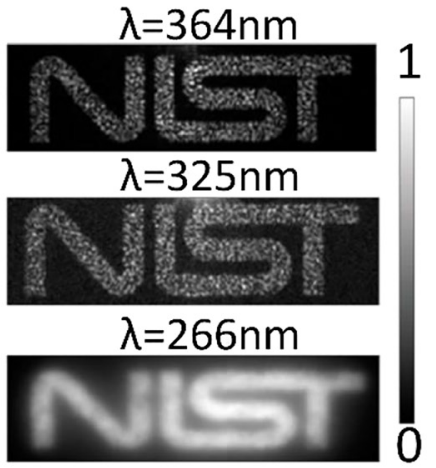

e

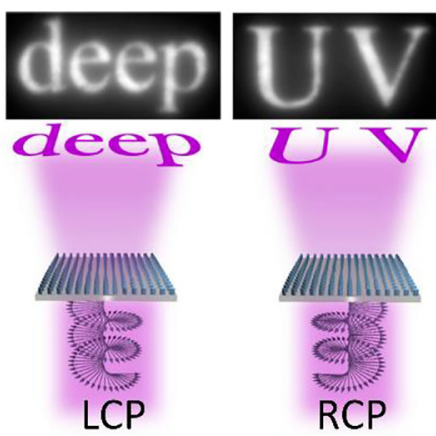

g

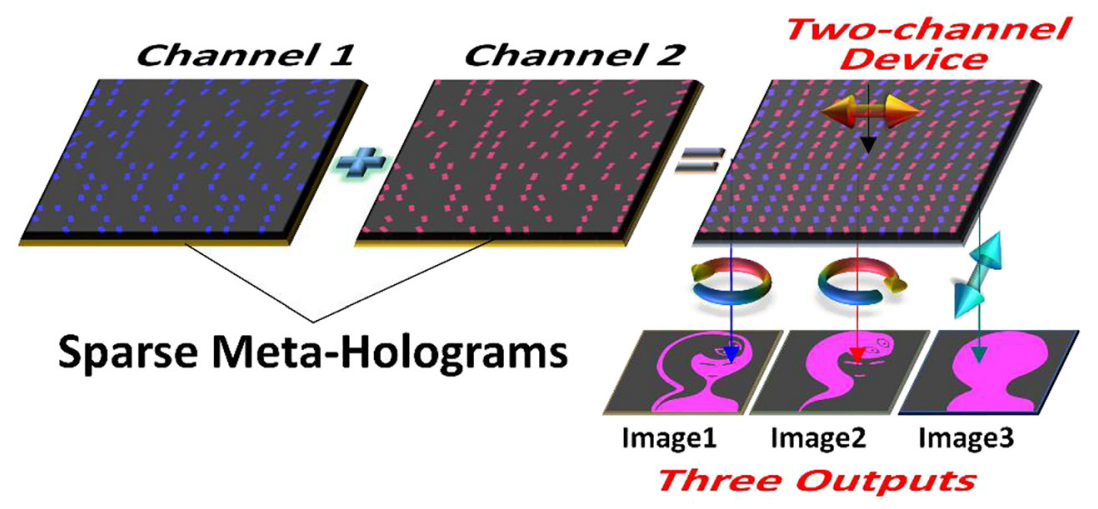

h

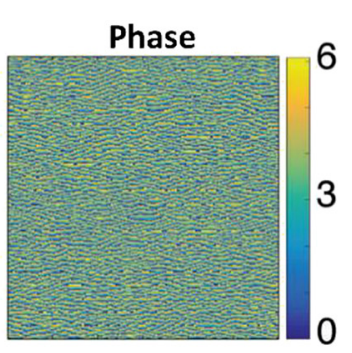

Image (Sim.)

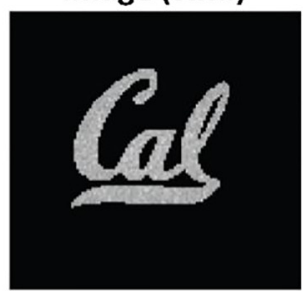

Image (Exp.)

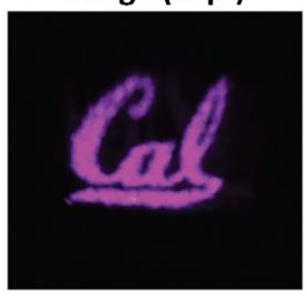

Exposed Photoresist Pattern

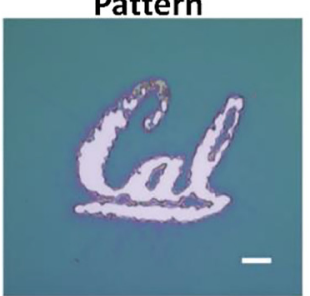

Figure 10: ( $a$ and b) Sketch (a) for the UV metasurface hologram and the experimental holographic image (b) by using the $\mathrm{Nb}_{2} \mathrm{O}_{5}$ nanobricks at the operating wavelength of $355 \mathrm{~nm}$. Huang et al. [109]. ( ) 2019, WILEY-VCH Verlag GmbH \& Co. KGaA, Weinheim. (c and d) Sketch (c) for the $\mathrm{HfO}_{2}$-based metahologram operating at the broadband wavelengths and their measured images (d). (e) Spin-multiplexed metahologram that reconstructs two spin-dependent images at the wavelength of $266 \mathrm{~nm}$. Zhang et al. [106]. Author copyright. (f) Spin-multiplexed UV metasurfaces encoding two sparse holograms that are used to create the complementary images of "girl" and "snake". (g) Experimental holographic image by labeling the polarization information where the blue color denotes the spin-up photons and the red for spin-down photons. Huang et al. [109]. @ 2019, WILEY-VCH Verlag GmbH \& Co. KGaA, Weinheim. (h) Si-based UV metahologram for lithography. The phase profile of the hologram is shown at the left panel. The simulated, experimental, and exposed images are provided at the right panel by addressing their names. Deng et al. [107]. (c) 2018 WILEY-VCH Verlag GmbH \& Co. KGaA, Weinheim. 
enhancing the homogeneity of the image. From the viewpoint of optical performance, the speckle is one of the main issues for the holography-based lithography. Except the dynamic random phase, one might remove the holography speckles by using a broadband laser source, or utilize advanced design of hologram phase via artificial intelligence technology. Reconfigurability of UV metasurfaces is another challenging problem for metaholography-based lithography [149].

\subsubsection{Photoemission spectroscopy by ultraviolet flat optics}

The high-energy photons of UV light could excite electron emission from materials, and is named as the photoelectric effect and discovered by Heinrich Hertz in 1887. By collecting the emitted electrons at the different angles, the electronic structure of solids can be probed by measuring the energy and momentum of the emitted electrons, which holds for the working principle of angle-resolved photoemission spectroscopy (ARPES) as a powerful tool for characterization of materials [150]. Traditional ARPES utilize X-ray light which is typically generated at expensive synchrotron radiation facilities. In addition, X-raybased ARPES have various drawbacks such as low energy resolution and bulky volume, which stimulated the invention of vacuum-UV-laser-based ARPES. Since the first development of laser ARPES in 2008 [151], several kinds of laser ARPES have been demonstrated at different laser wavelengths [152, 153]. The typical laser spots in these systems is $>3 \mu \mathrm{m}$, and therefore cannot satisfy the rapidly increasing requirement for characterizing micrometersized quantum materials, or two-dimensional materials. Very recently, a novel spherical-aberration-free zoneplate-based flat lens has been proposed to focus a $177 \mathrm{~nm}$ laser into a submicrometer spot of $\approx 0.8 \mu \mathrm{m}$ (Figure 11a-c) [108], which is the first demonstration of submicrometer resolved laser ARPES. Using this spot, various samples such as metallic gratings, graphene flakes are characterized with submicrometer resolution (Figure 11d and e). Although these zone plates are made of metallic materials for blocking light without electromagnetic resonances, they are categorized as the amplitude-type UV nanodevices, hereby within the scope of this review. In fact, at vacuum UV spectrum and beyond, absorption is the dominant electromagnetic loss mechanism and there are no transparent materials with high refractive index for development of these resonance-based metasurfaces. Thus, photonic platforms such as zone plates $[115,154-$ 157] and nanosieves [158-162] are good candidates for UV nanodevices. However, these platforms work by manipulating UV light's diffraction from the structures, where the strong diffraction (relative to the large ratio of the operating wavelength to the feature size of the structures) is a mandatory requirement for maintaining high optical performance of these devices. In addition, the diffraction of UV light also determines the efficiency of the entire device, and hence the structures for UV-light manipulation is extremely important.

\subsubsection{Nonlinear metaoptics}

As discussed above, nanostructures in metasurfaces could confine or induce electromagnetic resonance modes. Due to their subwavelength feature size, the electric fields within or near the nanostructures are much higher than the incident light, thus enhancing the light-matter interaction at UV wavelengths. Such a phenomenon has a direct application in creating UV light source through nonlinear processes, where the transfer efficiency of nonlinear signals is proportional to the intensity of the electric-field inside the nonlinear material. Categorized by the location of the induced optical modes, the reported works pursue two approaches (direct and indirect) to realize the creation of ultraviolent light.

The direct method is to use low-loss $\mathrm{ZnO}$ nanodisks for the confinement of a magnetic dipole induced by a circle electric displacement current (Figure 12a), such that the pumped $394 \mathrm{~nm}$ fs laser is trapped inside the nanostructures [116], with a transmission of nearly zero. Thus, the trapped optical modes interact with the second order nonlinearity of $\mathrm{ZnO}$ leading to second harmonic generation at $197 \mathrm{~nm}$ (Figure 12b). Meanwhile, due to the low absorption, these modes have long lifetime and therefore enable multiple resonances inside the nanostructures, eventually enhancing the nonlinear interactions. Alternatively, a better confinement of optical modes is achieved by employing bound states in the continuum (BIC), where the eigenmodes are localized in the nanostructures with a continuous spectrum of the radiating waves [163]. Due to a much narrower bandwidth, BICs have a longer lifetime and therefore should behave better for the generation of nonlinear signals, which, however, has not been demonstrated in experiment because BICs also need high reciprocity between both sides of the sample [164].

The other approach leverages an indirect interaction between a third material such as ITO and the induced confined optical modes that exist outside the metallic nanostructures. As shown in Figure 12c, the meta-atoms contain two "D"-like nanostructures that support the toroidal dipole resonance under the illumination of a linearly polarized light [43]. The toroidal dipole having a 


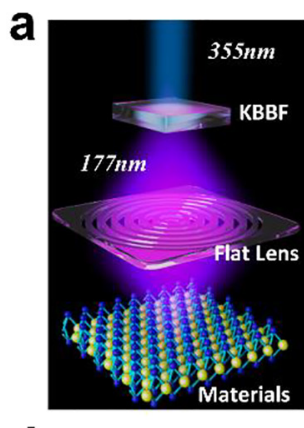

d

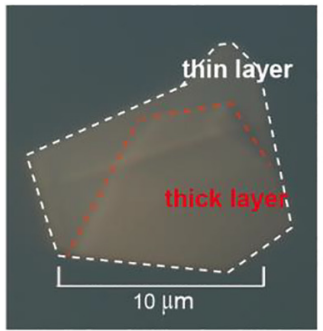

b

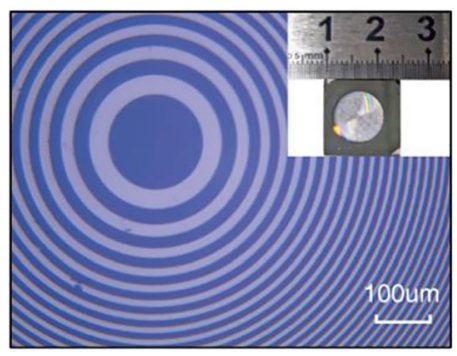

e

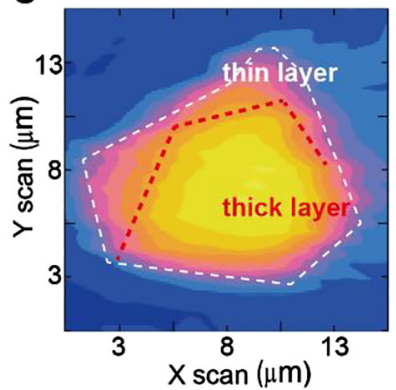

C

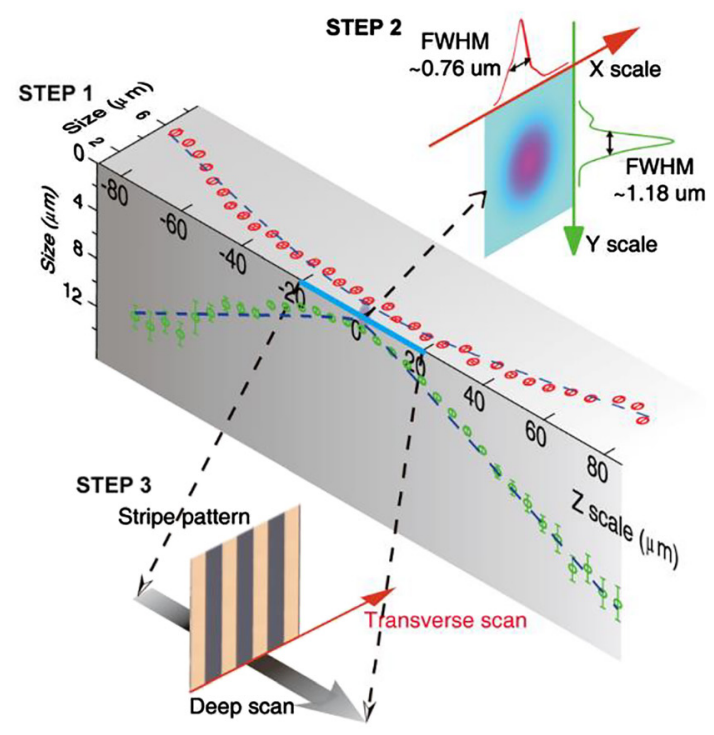

Figure 11: Photoemission spectroscopy with a submicrometer spot created by focusing the vacuum UV laser with a zone-plate flat lens. (a) Sketch of the spectroscopy. (b) Microscopy image of fabricated lens. (c) The experimental measured focal spot around the focal plane. (d-e) The microscopy (d) and scanned (e) images of the graphene flakes. The transmission of the scanned image can be used to calculate the layer number of the graphene flakes. Mao et al. [108]. Author copyright.

charge-current configuration exhibits strong electromagnetic localization, which can be used to enhance the nonlinear signals of the underlying ITO film. Under illumination with $785 \mathrm{~nm}$ wavelength fundamental laser, its third harmonic generation (THG) signals at $262 \mathrm{~nm}$ wavelength is $\approx 2.2$ times greater than that of the disk-dimer array, verifying the validity of the toroidal-dipole resonances (Figure 12d). In addition to enhancement of nonlinear signals, these dielectric metasurfaces with high-quality-factor nanoresonators can also be used to demonstrate UV circular dichroism [165].

\section{Outlook and conclusion}

Although UV nanophotonics has progressed rapidly through developments in various fields such as plasmonics, metamaterials, and metasurfaces over the past two decades, some fundamental challenges still exist which prevent UV nanophotonics from being widely employed for industrial applications. To pursue a better solution, we highlight some of these challenges below:

(i) UV-light sources are less developed with the intractable problems such as limited choice of operational wavelengths, poor tunability, low output power, and high cost (compared with their visible and infrared counterparts). Therefore, nonlinear process by pumping a nonlinear crystal with ultrafast laser is usually used to create the expected UV light [107], which, however, has usually bad coherence lengths and low power, making them nonideal for field applications. Another choice to obtain high brightness, monochromatic, and highly collimated UV light with even-shorter wavelengths is through synchrotron radiation sources, which are extremely high cost, bulky, and only available at very limited number of institutions worldwide.

(ii) Strong absorption of UV light limits the choice of materials to develop high-performance UV optical elements such as aberration-free lenses, high transmission, and ultrabroadband polarizers and waveplates. This material issue has much severe influence on UV nanophotonics that leverages electromagnetic responses based solely on electric and magnetic dipoles or multipoles, which further increase the loss of UV light. Moreover, to achieve the desired electromagnetic resonances, nanostructures in UV metasurfaces must have high refractive index and low absorption simultaneously, which are quite challenging to obtain for most natural materials. 
a

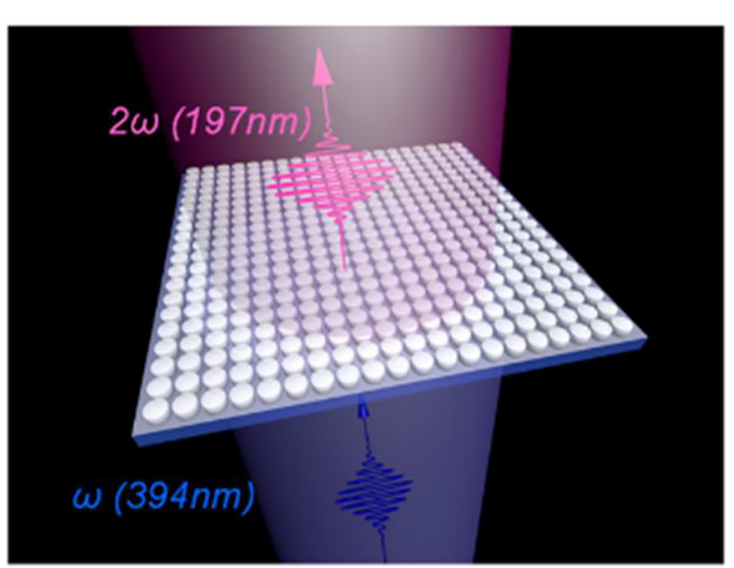

C

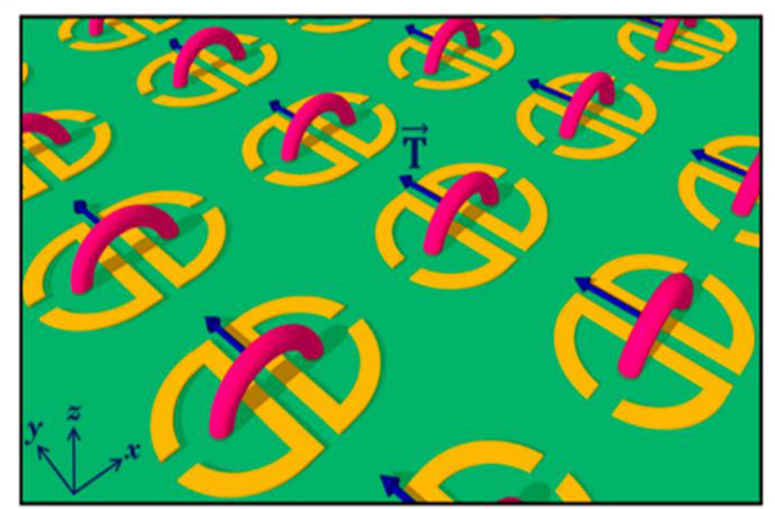

b

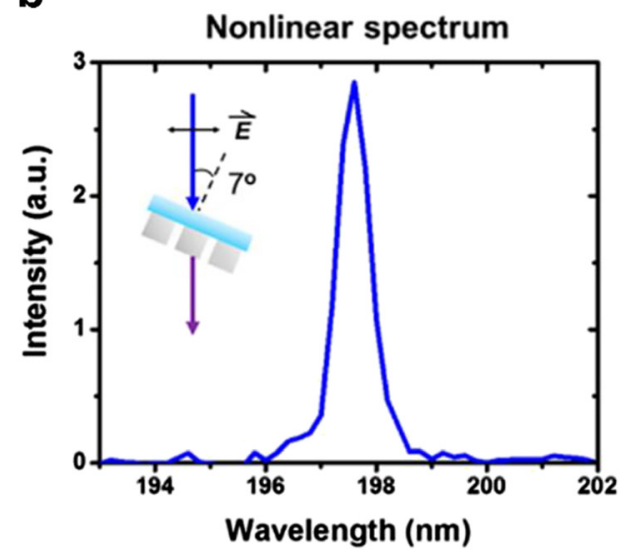

d

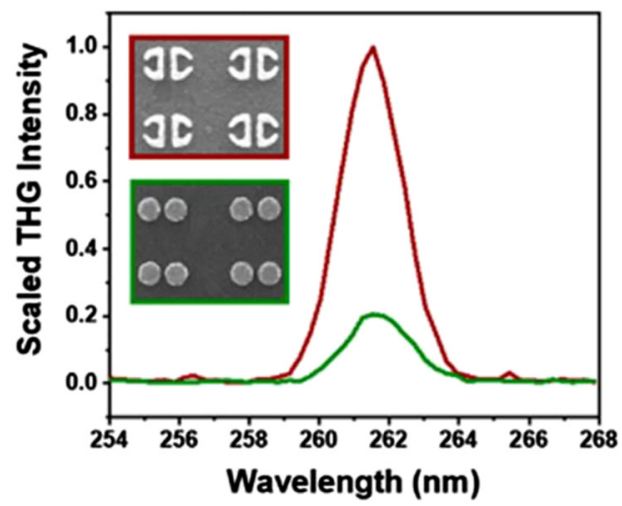

Figure 12: Nonlinear UV metasurfaces. (a and b) Second harmonic generation (a) by using the ZnO-based metasurfaces and the yielded SHG intensity (b). Semmlinger et al. [116]. ( 2018 American Chemical Society. (c) Third harmonic generation assisted by the toroidal dipole to enhance the THG signal (c). (d) A comparison between the THG signals created by the toroidal meta-atoms (red) and nanodisk (green) dimers. Ahmadivand et al. [43]. (c) 2019, American Chemical Society.

(iii) Challenges in fabrication become serious for shorterwavelength UV nanophotonics. As the wavelength scales down, the feature size of the nanostructures in the nanodevices must concurrently shrink, which has a twofold influence on fabrication. The first is a bad tolerance to the same fabrication error, and the other is that small features of nanostructures will eventually reach the limit of fabrication and cannot be realized experimentally. The current fabrication limits are $\approx 10 \mathrm{~nm}$ for electron-beam lithography [166], $\approx 50 \mathrm{~nm}$ for focused Ga-ion beam lithography [155], and $\approx 5 \mathrm{~nm}$ for focused Helium-ion beam lithography [167].

(iv) Detecting UV light efficiently is another challenge because of the low quantum efficiency for most UV detectors such as CMOS and CCD cameras. Most Si or GaN-based photodetectors have a quantum efficiency of $<30 \%$ in the near-UV range [168] and even lower at shorter wavelengths. It is caused by quite shallow penetration depth of UV photons inside the material of photodetectors due to strong absorption. Although amorphous-Se-based photodetectors have been reported with quantum efficiency more than $1000 \%$ at some special wavelengths $[169,170]$, any mature commercial products with high quantum efficiency over a broadband UV spectrum are still unavailable.

These challenges exist in the entire UV industry, and are not just limited to UV optics and nanophotonics. Considering the fact that traditional elements manipulate UV light via refraction and reflection for various functional devices, the emerging UV nanophotonics opens new opportunities, because it shapes UV light via diffraction at a subwavelength scale and can arbitrarily manipulate UV optics with advantages of dense integration, multifunctionality, wavelength multiplexing, light weight, and planar CMOS compatible fabrication. Together these advantages open unprecedented opportunities for the next-generation UV optics and might offer possible solutions to many of these ongoing challenges. Based on our previous knowledge and 
understanding of UV nanophotonics, we outline some potential future research directions.

First, developing new low-absorption materials for UV light is a long-term and persistent task by synthetizing novel inorganic or organic compound that might provide unexplored and large band structures for UV-light manipulation. Patterning artificial structures with few-nanometer feature size into a commonly used UV material or crystal [155] might modify optical responses of microscopic quasiparticles, such as acoustic and optical phonons, polaritons, excitons and orbitons, due to the redistributed density of electrons in material. A similar but different chemical method by selectively etching of target atoms and maintaining the others, named as "nanomesh" in chemistry [171, 172], is a good example to create a new material with distinct and unexplored electronic and optical properties. New UV materials will promote the development of UV elements, devices, and systems, as well as the investigation of UV lasers or diodes.

Second, the reported UV plasmonic and metasurface devices, such as lens, hologram, beam splitter, are still insufficient for boosting academic research and industrial applications relying on UV. Many important elements including waveplates, filters, polarizers, high-numericalaperture and aberration-free flat lenses, commercial UV detectors with high quantum efficiency, have not been demonstrated, hereby suggesting another future direction to substitute or compliment traditional bulk optics technology. Meanwhile, another possible engineering issue is to miniaturize the current UV bulky systems and lasers, which might enable portable UV products such as currency detectors and UV disinfection lighting devices.

Furthermore, UV nanophotonics at the even-shorter wavelengths such as extreme UV, vaccum UV and softX-ray does not exist due to the lack of commonly used optical elements. However, their extremely small wavelengths allow light-matter interaction with much higher precision for various applications in nuclear physics, semiconductor lithography, and material analysis. Therefore, it is expected that VUV and EUV nanophotonics will be the future once the auxiliary optics can be easily obtained.

Finally, searching for new applications in multidisciplinary fields is one future goal of UV industry. The already developed applications should be improved further, for example, high-efficiency and ultrabroadband UV metalenses with the intriguing properties including subdiffraction limit focusing and imaging, increasing the security level of UV holography by using more degrees of freedom for the purpose of information coding and anticounterfeiting, suppressing the focusing spot of UV light in photoemission spectroscopy down to $100 \mathrm{~nm}$ that is comparable to that of X-ray-based ARPES, developing novel nanostructures with stronger nonlinear responses via emerging physical mechanisms such as bound states in the continuum [173], epsilon-zero materials [9], and Moire lattices [174]. New applications of UV nanophotonics might occur if it links communication [175], photocatalysis [176, 177], optogenetics [178] and oxidation [179]. In addition, taking into account that the bandgaps of DNAs and proteins are located at the UV spectrum [180], the UV-nanophotonics-based biosensor and biodetection might become next exciting application in combination with Raman scattering, nonlinear harmonic generation or photoluminescence.

In conclusion, we have given a brief introduction and overview of UV nanophotonics. The recent advances in UV plasmonics, metamaterials, and metasurfaces have been reviewed along with discussions of their physical origins, unique properties, existing problems, and demonstrated applications. The challenges of efficient and bright light sources, material absorption, device fabrication, light detection, and correct usage in practical applications are discussed in detail with the hope of a solution in the near future. The prospects of future research directions and unsolved problems has been provided on the basis of our best knowledge of the field of UV optics and nanophotonics. Although UV nanophotonics has gained increasing attention with exciting achievements recently, it is still at its infancy in both scientific research and industrial applications and therefore it is expected to develop further in the future due to their manyfold advantages in light-matter interaction.

Author contributions: All the authors have accepted responsibility for the entire content of this submitted manuscript and approved submission.

Research funding: K.H. thanks CAS Pioneer Hundred Talents Program, "the Fundamental Research Funds for the Central Universities" in China, USTC Research Funds of the Double First-Class Initiative (Grant No. YD2030002003), the National Natural Science Foundation of China (Grant Nos. 61875181 and 61705085), and the support from the University of Science and Technology of China's Centre for Micro and Nanoscale Research and Fabrication. C.Z. and Z.L. acknowledge the startup funding from Huazhong University of Science and Technology and support from the National Natural Science Foundation of China (Grant No. 62075078). T.X. acknowledges support from the National Natural Science Foundation of China (Grant No. 11774163). W.Z. and A.A. acknowledge support under the Cooperative Research Agreement between the University of Maryland and the National Institute of Standards and Technology 
Physical Measurement Laboratory, Award No. 70NANB14H209, through the University of Maryland.

Conflict of interest statement: The authors declare no conflicts of interest regarding this article.

\section{References}

[1] J. Barth, "Johann Wilhelm Ritter (1776-1810) and the discovery of ultraviolet irradiation 185 years ago," Der Hautarzt; Zeitschrift fur Dermatologie, Venerologie, und verwandte Gebiete, vol. 38, pp. 301-303, 1987.

[2] C. Alapetite, "Use of the alkaline comet assay to detect DNA repair deficiencies in human fibroblasts exposed to UVC, UVB, UVA and gamma-rays," Int. J. Radiat. Biol., vol. 69, pp. 359-369, 1996.

[3] M. S. Shur and R. Gaska, "Deep-ultraviolet light-emitting diodes," IEEE Trans. Electron. Dev., vol. 57, pp. 12-25, 2010.

[4] A. Khan, K. Balakrishnan, and T. Katona, "Ultraviolet lightemitting diodes based on group three nitrides," Nat. Photonics, vol. 2, pp. 77-84, 2008.

[5] K. H. Li, X. Liu, Q. Wang, et al., "Ultralow-threshold electrically injected AlGaN nanowire ultraviolet lasers on Si operating at low temperature," Nat. Nanotechnol., vol. 10, pp. 140-144, 2015.

[6] H. Wu, H. Yu, Z. Yang, et al., "Designing a deep-ultraviolet nonlinear optical material with a large second harmonic generation response," J. Am. Chem. Soc., vol. 135, pp. 4215-4218, 2013.

[7] T. T. Luu, Z. Yin, A. Jain, et al., "Extreme-ultraviolet highharmonic generation in liquids," Nat. Commun., vol. 9, p. 3723, 2018.

[8] S. Ghimire, A. D. DiChiara, E. Sistrunk, et al., "Observation of high-order harmonic generation in a bulk crystal," Nat. Phys., vol. 7, pp. 138-141, 2011.

[9] Y. Yang, J. Lu, A. Manjavacas, et al., "High-harmonic generation from an epsilon-near-zero material," Nat. Phys., vol. 15, pp. 1022-1026, 2019.

[10] B. Wu, D. Tang, N. Ye, et al., "Linear and nonlinear optical properties of the $\mathrm{KBe}_{2} \mathrm{BO}_{3} \mathrm{~F}_{2}$ (KBBF) crystal," Opt. Mater., vol. 5, pp. 105-109, 1996.

[11] G. Zou, N. Ye, L. Huang, et al., "Alkaline-alkaline earth fluoride carbonate crystals $\mathrm{ABCO}_{3} \mathrm{~F}(\mathrm{~A}=\mathrm{K}, \mathrm{Rb}, \mathrm{Cs} ; \mathrm{B}=\mathrm{Ca}, \mathrm{Sr}, \mathrm{Ba})$ as nonlinear optical materials," J. Am. Chem. Soc., vol. 133, pp. 20001-20007, 2011.

[12] R. Schupp, F. Torretti, R. Meijer, et al., "Efficient generation of extreme ultraviolet light from Nd: YAG-driven microdroplet-tin plasma," Phys. Rev. Appl., vol. 12, p. 014010, 2019.

[13] T. Shintake, H. Tanaka, T. Hara, et al., “A compact free-electron laser for generating coherent radiation in the extreme ultraviolet region," Nat. Photonics, vol. 2, pp. 555-559, 2008.

[14] N. J. Greenfield, "Using circular dichroism spectra to estimate protein secondary structure," Nat. Protoc., vol. 1, pp. 2876-2890, 2006.

[15] M. Oppermann, B. Bauer, T. Rossi, et al., "Ultrafast broadband circular dichroism in the deep ultraviolet," Optica, vol. 6, pp. 56-60, 2019.

[16] B. Ranjbar and P. Gill, "Circular dichroism techniques: biomolecular and nanostructural analyses - a review," Chem. Biol. Drug Des., vol. 74, pp. 101-120, 2009.
[17] A. O. Nwokeoji, P. M. Kilby, D. E. Portwood, et al., "Accurate quantification of nucleic acids using hypochromicity measurements in conjunction with UV spectrophotometry," Anal. Chem., vol. 89, pp. 13567-13574, 2017.

[18] A. Dell'Anno, M. Fabiano, G. C. A. Duineveld, et al., "Nucleic acid (DNA, RNA) quantification and RNA/DNA ratio determination in marine sediments: comparison of spectrophotometric, fluorometric, and HighPerformance liquid chromatography methods and estimation of detrital DNA," Appl. Environ. Microbiol., vol. 64, pp. 3238-3245, 1998.

[19] R. H. Stulen and D. W. Sweeney, "Extreme ultraviolet lithography,” IEEE J. Quant. Electron., vol. 35, pp. 694-699, 1999.

[20] B. Wu and A. Kumar, "Extreme ultraviolet lithography: a review," J. Vac. Sci. Technol. B Microelectron. Nanometer Struct. Process. Meas. Phenom., vol. 25, pp. 1743-1761, 2007.

[21] M. Heßling, K. Hönes, P. Vatter, et al., "Ultraviolet irradiation doses for coronavirus inactivation - review and analysis of coronavirus photoinactivation studies," GMS Hyg. Infect. Contr., vol. 15, p. Doc08, 2020.

[22] T. Bintsis, E. Litopoulou-Tzanetaki, and R. K. Robinson, "Existing and potential applications of ultraviolet light in the food industry - a critical review," J. Sci. Food Agric., vol. 80, pp. 637-645, 2000.

[23] Y. Kivshar, "All-dielectric meta-optics and non-linear nanophotonics," Natl. Sci. Rev, vol. 5, pp. 144-158, 2018.

[24] M. Pu, X. Ma, X. Li, et al., "Merging plasmonics and metamaterials by two-dimensional subwavelength structures," J. Mater. Chem. C, vol. 5, pp. 4361-4378, 2017.

[25] A. M. Urbas, Z. Jacob, L. D. Negro, et al., "Roadmap on optical metamaterials,” J. Opt., vol. 18, p. 093005, 2016.

[26] K. Yao and Y. Liu, "Plasmonic metamaterials," Nanotechnol. Rev., vol. 3, pp. 177-210, 2014.

[27] X. Luo, "Engineering optics 2.0: a revolution in optical materials, devices, and systems," ACS Photonics, vol. 5, pp. 4724-4738, 2018.

[28] Y. Liu and X. Zhang, "Metamaterials: a new Frontier of science and technology," Chem. Soc. Rev., vol. 40, pp. 2494-2507, 2011.

[29] A. Karabchevsky, A. Katiyi, A. S. Ang, et al., "On-chip nanophotonics and future challenges," Nanophotonics, vol. 9, pp. 3733-3753, 2020.

[30] S. A. Maier, Plasmonics: Fundamentals and Applications, New York, Springer Science \& Business Media, 2007.

[31] A. V. Zayats, I. I. Smolyaninov, and A. A. Maradudin, "Nanooptics of surface plasmon polaritons," Phys. Rep., vol. 408, pp. 131-314, 2005.

[32] C. Zhang, N. Kinsey, L. Chen, et al., "High-performance doped silver films: overcoming fundamental material limits for nanophotonic applications," Adv. Mater., vol. 29, no. 19, p. 1605177, 2018.

[33] R. Lemasters, C. Zhang, M. Manjare, et al., "Ultrathin wetting layer-free plasmonic gold films," ACS Photonics, vol. 6, no. 11, pp. 2600-2606, 2019.

[34] C. Langhammer, M. Schwind, B. Kasemo, et al., "Localized surface plasmon resonances in aluminum nanodisks," Nano Lett., vol. 8, pp. 1461-1471, 2008.

[35] J. M. McMahon, G. C. Schatz, and S. K. Gray, "Plasmonics in the ultraviolet with the poor metals Al, $\mathrm{Ga}, \mathrm{In}, \mathrm{Sn}, \mathrm{Tl}, \mathrm{Pb}$, and $\mathrm{Bi}$," Phys. Chem. Chem. Phys., vol. 15, pp. 5415-5423, 2013. 
[36] P. Shekhar, S. Pendharker, H. Sahasrabudhe, et al., "Extreme ultraviolet plasmonics and Cherenkov radiation in silicon," Optica, vol. 5, pp. 1590-1596, 2018.

[37] Z. Dong, T. Wang, X. Chi, et al., "Ultraviolet interband plasmonics with Si nanostructures," Nano Lett., vol. 19, pp. 8040-8048, 2019.

[38] M. W. Knight, L. Liu, Y. Wang, et al., “Aluminum plasmonic nanoantennas," Nano Lett., vol. 12, pp. 6000-6004, 2012.

[39] J. B. Khurgin, "How to deal with the loss in plasmonics and metamaterials," Nat. Nanotechnol., vol. 10, pp. 2-6, 2015.

[40] I. Gryczynski, J. Malicka, Z. Gryczynski, et al., "Ultraviolet surface plasmon-coupled emission using thin aluminum films," Anal. Chem., vol. 76, pp. 4076-4081, 2004.

[41] F. J. García de Abajo, "Optical excitations in electron microscopy,” Rev. Mod. Phys., vol. 82, pp. 209-275, 2010.

[42] B. Ren, X. F. Lin, Z. L. Yang, et al., "Surface-enhanced Raman scattering in the ultraviolet spectral region: UV-SERS on rhodium and ruthenium electrodes," J. Am. Chem. Soc., vol. 125, pp. 9598-9, 2003.

[43] A. Ahmadivand, M. Semmlinger, L. Dong, et al., "Toroidal dipoleenhanced third harmonic generation of deep ultraviolet light using plasmonic meta-atoms," Nano Lett., vol. 19, pp. 605-611, 2018.

[44] D. Li, X. Sun, H. Song, et al., "Realization of a high-performance GaN UV detector by nanoplasmonic enhancement," Adv. Mater., vol. 24, pp. 845-849, 2012.

[45] V. G. Veselago, "The electrodynamics of substances with simultaneously negative values of epsilon and $\mu$," Sov. Phys. Usp., vol. 10, pp. 509-514, 1968.

[46] S. Xi, H. Chen, T. Jiang, et al., "Experimental verification of reversed Cherenkov radiation in left-handed metamaterial," Phys. Rev. Lett., vol. 103, p. 194801, 2009.

[47] R. A. Shelby, D. R. Smith, and S. Schultz, "Experimental verification of a negative index of refraction," Science, vol. 292, pp. 77-79, 2001.

[48] J. B. Pendry, "Negative refraction makes a perfect lens," Phys. Rev. Lett., vol. 85, p. 3966, 2000.

[49] D. Ye, K. Chang, L. Ran, et al., "Microwave gain medium with negative refractive index," Nat. Commun., vol. 5, p. 5841, 2014.

[50] V. M. Shalaev, "Optical negative-index metamaterials," Nat. Photonics, vol. 1, pp. 41-48, 2007.

[51] H. J. Lezec, J. A. Dionne, and H. A. Atwater, "Negative refraction at visible frequencies," Science, vol. 316, pp. 430-432, 2007.

[52] E. Verhagen, R. de Waele, L. Kuipers, et al., "Three-dimensional negative index of refraction at optical frequencies by coupling plasmonic waveguides," Phys. Rev. Lett., vol. 105, p. 223901, 2010.

[53] T. Xu, A. Agrawal, M. Abashin, et al., "All-angle negative refraction and active flat lensing of ultraviolet light," Nature, vol. 497, pp. 470-474, 2013.

[54] A. Poddubny, I. Iorsh, P. Belov, et al., "Hyperbolic metamaterials," Nat. Photonics, vol. 7, pp. 948-957, 2013.

[55] C. Zhang, N. Hong, C. Ji, et al., "Robust extraction of hyperbolic metamaterial permittivity using total internal reflection ellipsometry," ACS Photonics, vol. 5, pp. 2234-2242, 2018.

[56] P. Shekhar, J. Atkinson, and Z. Jacob, “Hyperbolic metamaterials: fundamentals and applications," Nano Convergence, vol. 1, p. 14, 2014.
[57] X. Yang, J. Yao, J. Rho, et al., "Experimental realization of threedimensional indefinite cavities at the nanoscale with anomalous scaling laws," Nat. Photonics, vol. 6, pp. 450-454, 2012.

[58] H. N. S. Krishnamoorthy, Z. Jacob, E. Narimanov, et al., “Topological transitions in metamaterials," Science, vol. 336, pp. 205-209, 2012.

[59] D. Lu, J. J. Kan, E. E. Fullerton, et al., "Enhancing spontaneous emission rates of molecules using nanopatterned multilayer hyperbolic metamaterials," Nat. Nanotechnol., vol. 9, pp. 48-53, 2014.

[60] K.-C. Shen, C.-T. Ku, C. Hsieh, et al., "Deep-ultraviolet hyperbolic metacavity laser," Adv. Mater., vol. 30, p. 1706918, 2018.

[61] Y. Guo, C. L. Cortes, S. Molesky, et al., "Broadband superPlanckian thermal emission from hyperbolic metamaterials," Appl. Phys. Lett., vol. 101, p. 131106, 2012.

[62] P. N. Dyachenko, S. Molesky, A. Y. Petrov, et al., "Controlling thermal emission with refractory epsilon-near-zero metamaterials via topological transitions," Nat. Commun., vol. 7, p. 11809, 2016.

[63] T. U. Tumkur, L. Gu, J. K. Kitur, et al., "Control of absorption with hyperbolic metamaterials,” Appl. Phys. Lett., vol. 100, p. 161103, 2012.

[64] Z. Liu, H. Lee, Y. Xiong, et al., "Far-field optical hyperlens magnifying sub-diffraction-limited objects," Science, vol. 315, p. 1686, 2007.

[65] X. Chen, C. Zhang, F. Yang, et al., "Plasmonic lithography utilizing epsilon near zero hyperbolic metamaterial,” ACS Nano, vol. 11, pp. 9863-9868, 2017.

[66] T. Xu and H. J. Lezec, "Visible-frequency asymmetric transmission devices incorporating a hyperbolic metamaterial," Nat. Commun., vol. 5, p. 4141, 2014.

[67] N. Yu, P. Genevet, M. A. Kats, et al., "Light propagation with phase discontinuities: generalized laws of reflection and refraction," Science, vol. 334, pp. 333-337, 2011.

[68] P. Lalanne, S. Astilean, P. Chavel, et al., "Blazed binary subwavelength gratings with efficiencies larger than those of conventional Échelette gratings," Opt. Lett., vol. 23, pp. 1081-1083, 1998.

[69] Z. Bomzon, G. Biener, V. Kleiner, et al., "Spatial Fouriertransform polarimetry using space-variant subwavelength metal-stripe polarizers," Opt. Lett., vol. 26, pp. 1711-1713, 2001.

[70] K. Huang, D. Zhao, F. Tjiptoharsono, et al., "Bio-inspired photonic masquerade with perturbative metasurfaces," ACS Nano, vol. 14, pp. 7529-7537, 2020.

[71] K. Huang, H. Liu, S. Restuccia, et al., "Spiniform phase-encoded metagratings entangling arbitrary rational-order orbital angular momentum," Light Sci. Appl., vol. 7, p. 17156, 2018.

[72] T. Stav, A. Faerman, E. Maguid, et al., "Quantum entanglement of the spin and orbital angular momentum of photons using metamaterials," Science, vol. 361, pp. 1101-1104, 2018.

[73] K. Wang, J. G. Titchener, S. S. Kruk, et al., "Quantum metasurface for multiphoton interference and state reconstruction," Science, vol. 361, pp. 1104-1108, 2018.

[74] F. Yesilkoy, E. R. Arvelo, Y. Jahani, et al., "Ultrasensitive hyperspectral imaging and biodetection enabled by dielectric metasurfaces," Nat. Photonics, vol. 13, pp. 390-396, 2019.

[75] D. Lin, M. Melli, E. Poliakov, et al., "Optical metasurfaces for high angle steering at visible wavelengths," Sci. Rep., vol. 7, p. 2286, 2017. 
[76] T. Phan, D. Sell, E. W. Wang, et al., "High-efficiency, large-area, topology-optimized metasurfaces," Light Sci. Appl., vol. 8, p. 48, 2019.

[77] M. Khorasaninejad, W. T. Chen, R. C. Devlin, et al., "Metalenses at visible wavelengths: diffraction-limited focusing and subwavelength resolution imaging," Science, vol. 352, pp. 1190-1194, 2016.

[78] R. J. Lin, V.-C. Su, S. Wang, et al., "Achromatic metalens array for full-colour light-field imaging,” Nat. Nanotechnol., vol. 14, pp. 227-231, 2019.

[79] W. Yang, S. Xiao, Q. Song, et al., "All-dielectric metasurface for high-performance structural color," Nat. Commun., vol. 11, p. 1864, 2020.

[80] S. Sun, Z. Zhou, C. Zhang, et al., "All-dielectric full-color printing with $\mathrm{TiO}_{2}$ metasurfaces," ACS Nano, vol. 11, pp. 4445-4452, 2017.

[81] B. Yang, W. Liu, Z. Li, et al., "Ultrahighly saturated structural colors enhanced by multipolar-modulated metasurfaces," Nano Lett., vol. 19, pp. 4221-4228, 2019.

[82] W.-J. Joo, J. Kyoung, M. Esfandyarpour, et al., "Metasurfacedriven OLED displays beyond 10,000 pixels per inch," Science, vol. 370, pp. 459-463, 2020.

[83] Y.-K. R. Wu, A. E. Hollowell, C. Zhang, et al., “Angle-insensitive structural colours based on metallic nanocavities and coloured pixels beyond the diffraction limit," Sci. Rep., vol. 3, p. 1194, 2013.

[84] L. Huang, S. Zhang, and T. Zentgraf, "Metasurface holography: from fundamentals to applications," Nanophotonics, vol. 7, pp. 1169-1190, 2018.

[85] A. C. Overvig, S. Shrestha, S. C. Malek, et al., "Dielectric metasurfaces for complete and independent control of the optical amplitude and phase," Light Sci. Appl., vol. 8, p. 92, 2019.

[86] Q. Fan, M. Liu, C. Zhang, et al., "Independent amplitude control of arbitrary orthogonal states of polarization via dielectric metasurfaces," Phys. Rev. Lett., vol. 125, p. 267402, 2020.

[87] G. Zheng, H. Mühlenbernd, M. Kenney, et al., "Metasurface holograms reaching $80 \%$ efficiency," Nat. Nanotechnol., vol. 10, pp. 308-312, 2015.

[88] Q. Fan, W. Zhu, Y. Liang, et al., "Broadband generation of photonic spin-controlled arbitrary accelerating light beams in the visible," Nano Lett., vol. 19, pp. 1158-1165, 2019.

[89] W. T. Chen, M. Khorasaninejad, A. Y. Zhu, et al., "Generation of wavelength-independent subwavelength Bessel beams using metasurfaces," Light Sci. Appl., vol. 6, p. e16259, 2017.

[90] C. Zhang, C. Pfeiffer, T. Jang, et al., “Breaking Malus' law: highly efficient, broadband, and angular robust asymmetric light transmitting metasurface," Laser Photonics Rev., vol. 10, pp. 791-798, 2016.

[91] K. Chen, G. Ding, G. Hu, et al., "Directional janus metasurface," Adv. Mater., vol. 32, p. 1906352, 2020.

[92] D. Ma, Z. Li, Y. Zhang, et al., "Giant spin-selective asymmetric transmission in multipolar-modulated metasurfaces," Opt. Lett., vol. 44, pp. 3805-3808, 2019.

[93] S. Divitt, W. Zhu, C. Zhang, et al., "Ultrafast optical pulse shaping using dielectric metasurfaces," Science, vol. 364, pp. 890-894, 2019.

[94] A. M. Shaltout, K. G. Lagoudakis, J. van de Groep, et al., "Spatiotemporal light control with frequency-gradient metasurfaces," Science, vol. 365, pp. 374-377, 2019.

[95] A. Arbabi, Y. Horie, M. Bagheri, et al., "Dielectric metasurfaces for complete control of phase and polarization with subwavelength spatial resolution and high transmission," Nat. Nanotechnol., vol. 10, pp. 937-943, 2015.

[96] K. Huang, Z. Dong, S. Mei, et al., "Silicon multi-meta-holograms for the broadband visible light," Laser Photonics Rev., vol. 10, pp. 500-509, 2016.

[97] E. Hasman, V. Kleiner, G. Biener, et al., "Polarization dependent focusing lens by use of quantized Pancharatnam-Berry phase diffractive optics," Appl. Phys. Lett., vol. 82, pp. 328-330, 2003.

[98] M. Decker, I. Staude, M. Falkner, et al., "High-efficiency dielectric Huygens' surfaces,” Adv. Opt. Mater, vol. 3, pp. 813-820, 2015.

[99] L. Zhang, S. Mei, K. Huang, et al., "Advances in full control of electromagnetic waves with metasurfaces," Adv. Opt. Mater., vol. 4, pp. 818-833, 2016.

[100] H.-T. Chen, A. J. Taylor, and N. Yu, "A review of metasurfaces: physics and applications," Rep. Prog. Phys., vol. 79, p. 076401, 2016.

[101] S. B. Glybovski, S. A. Tretyakov, P. A. Belov, et al., "Metasurfaces: from microwaves to visible," Phys. Rep., vol. 634, pp. 1-72, 2016.

[102] S. M. Kamali, E. Arbabi, A. Arbabi, et al., "A review of dielectric optical metasurfaces for wavefront control," Nanophotonics, vol. 7, pp. 1041-1068, 2018.

[103] N. Yu and F. Capasso, "Flat optics with designer metasurfaces," Nat. Mater., vol. 13, pp. 139-150, 2014.

[104] P. Genevet and F. Capasso, "Holographic optical metasurfaces: a review of current progress," Rep. Prog. Phys., vol. 78, p. $024401,2015$.

[105] D. Attwood and A. Sakdinawat, $X$-Rays and Extreme Ultraviolet Radiation: Principles and Applications, Cambridge, Cambridge University Press, 2017.

[106] C. Zhang, S. Divitt, Q. Fan, et al., "Low-loss metasurface optics down to the deep ultraviolet region," Light Sci. Appl., vol. 9, pp. 1-10, 2020.

[107] Y. Deng, X. Wang, Z. Gong, et al., “All-silicon broadband ultraviolet metasurfaces,” Adv. Mater., vol. 30, p. 1802632, 2018.

[108] Y. Mao, D. Zhao, S. Yan, et al., "A vacuum ultraviolet laser with a submicrometer spot for spatially resolved photoemission spectroscopy," Light Sci. Appl., vol. 10, p. 22, 2021.

[109] K. Huang, J. Deng, H. S. Leong, et al., "Ultraviolet metasurfaces of $\approx 80 \%$ efficiency with antiferromagnetic resonances for optical vectorial anti-counterfeiting," Laser Photonics Rev., vol. 13, p. 1800289, 2019.

[110] O. Hemmatyar, S. Abdollahramezani, Y. Kiarashinejad, et al., "Full color generation with fano-type resonant $\mathrm{HfO}_{2}$ nanopillars designed by a deep-learning approach," Nanoscale, vol. 11, pp. 21266-21274, 2019.

[111] M. Khorasaninejad and K. B. Crozier, "Silicon nanofin grating as a miniature chirality-distinguishing beam-splitter," Nat. Commun., vol. 5, p. 5386, 2014.

[112] W. T. Chen, A. Y. Zhu, V. Sanjeev, et al., "A broadband achromatic metalens for focusing and imaging in the visible," Nat. Nanotechnol., vol. 13, pp. 220-226, 2018.

[113] S. Wang, P. C. Wu, V.-C. Su, et al., "A broadband achromatic metalens in the visible," Nat. Nanotechnol., vol. 13, pp. 227-232, 2018.

[114] P. Lalanne and P. Chavel, "Metalenses at visible wavelengths: past, present, perspectives," Laser Photonics Rev., vol. 11, p. 1600295, 2017. 
[115] K. Huang, F. Qin, Hong Liu, et al., "Planar diffractive lenses: fundamentals, functionalities, and applications," Adv. Mater., vol. 30, p. 1704556, 2018.

[116] M. Semmlinger, M. L. Tseng, J. Yang, et al., "Vacuum ultraviolet light-generating metasurface," Nano Lett., vol. 18, pp. 5738-5743, 2018.

[117] L. Huang, X. Chen, H. Mühlenbernd, et al., "Dispersionless phase discontinuities for controlling light propagation," Nano Lett., vol. 12, pp. 5750-5755, 2012.

[118] M. Mehmood, S. Mei, S. Hussain, et al., "Visible-frequency metasurface for structuring and spatially multiplexing optical vortices," Adv. Mater., vol. 28, pp. 2533-2539, 2016.

[119] A. Niv, G. Biener, V. Kleiner, et al., "Manipulation of the Pancharatnam phase in vectorial vortices," Opt. Express, vol. 14, pp. 4208-4220, 2006.

[120] W. Luo, S. Sun, H.-X. Xu, et al., "Transmissive ultrathin Pancharatnam-Berry metasurfaces with nearly $100 \%$ efficiency," Phys. Rev. Appl., vol. 7, p. 044033, 2017.

[121] Y. F. Yu, A. Y. Zhu, R. Paniagua-Domínguez, et al., "Hightransmission dielectric metasurface with $2 \pi$ phase control at visible wavelengths," Laser Photonics Rev., vol. 9, pp. 412-418, 2015.

[122] J. Li, G. Si, H. Liu, et al., "Resonance-free ultraviolet metaoptics via photon nanosieves,” Opt. Lett., vol. 44, pp. 3418-3421, 2019.

[123] L. Drescher, O. Kornilov, T. Witting, et al., "Extreme-ultraviolet refractive optics," Nature, vol. 564, pp. 91-94, 2018.

[124] S. Sun, K.-Y. Yang, C.-M. Wang, et al., "High-efficiency broadband anomalous reflection by gradient meta-surfaces," Nano Lett., vol. 12, pp. 6223-6229, 2012.

[125] Y. Ra'di, D. L. Sounas, and A. Alu, “Metagratings: beyond the limits of graded metasurfaces for wave front control," Phys. Rev. Lett., vol. 119, p. 067404, 2017.

[126] A. Yulaev, W. Zhu, C. Zhang, et al., "Metasurface-integrated photonic platform for versatile free-space beam projection with polarization control," ACS Photonics, vol. 6, pp. 2902-2909, 2019.

[127] N. Mahmood, M. Q. Mehmood, and F. A. Tahir, "Diamond stepindex nanowaveguide to structure light efficiently in near and deep ultraviolet regimes," Sci. Rep., vol. 10, pp. 1-10, 2020.

[128] L. Guo, S. Xu, R. Wan, et al., "Design of aluminum nitride metalens in the ultraviolet spectrum," J. Nanophotonics, vol. 12, p. 043513, 2018

[129] F. Matusalem, M. Marques, L. K. Teles, et al., "Electronic properties of fluorides by efficient approximated quasiparticle DFT-1/2 and PSIC methods: $\mathrm{BaF}_{2}, \mathrm{CaF}_{2}$ and $\mathrm{CdF}_{2}$ as test cases," J. Phys. Condens. Matter., vol. 30, p. 365501, 2018.

[130] I. H. Malitson, "Interspecimen comparison of the refractive index of fused silica," Josa, vol. 55, pp. 1205-1209, 1965

[131] M. J. Dodge, "Refractive properties of magnesium fluoride," Appl. Opt., vol. 23, pp. 1980-1985, 1984.

[132] D. Li, K. Jiang, X. Sun, et al., "AlGaN photonics: recent advances in materials and ultraviolet devices," Adv. Opt. Photonics, vol. 10, pp. 43-110, 2018.

[133] C. Xie, X.-T. Lu, X.-W. Tong, et al., "Recent progress in solarblind deep-ultraviolet photodetectors based on inorganic ultrawide bandgap semiconductors," Adv. Funct. Mater., vol. 29, p. 1806006, 2019.

[134] D.-B. Li, X.-J. Sun, Y.-P. Jia, et al., "Direct observation of localized surface plasmon field enhancement by Kelvin probe force microscopy," Light Sci. Appl., vol. 6, pp. e17038, 2017.
[135] G. Bao, D. Li, X. Sun, et al., "Enhanced spectral response of an AlGaN-based solar-blind ultraviolet photodetector with Al nanoparticles," Opt. Express, vol. 22, pp. 24286-24293, 2014.

[136] Y. Wu, X.-J. Sun, Y.-P. Jia, et al., "Review of improved spectral response of ultraviolet photodetectors by surface plasmon," Chin. Phys. B, vol. 27, p. 126101, 2018.

[137] K. Kneipp, M. Moskovits, and H. Kneipp, Surface-Enhanced Raman Scattering: Physics and Applications, vol. 103, Berlin, Springer, 2006

[138] R. Wen and Y. Fang, "Surface Raman scattering studies on the adsorption of p-hydroxybenzoic acid at Au electrodes with ultraviolet excitation," J. Electroanal. Chem., vol. 576, pp. 237-242, 2005.

[139] T. Dörfer, M. Schmitt, and J. Popp, “Deep-UV surface-enhanced Raman scattering," J. Raman Spectrosc., vol. 38, pp. 1379-1382, 2007.

[140] A. Taguchi, N. Hayazawa, K. Furusawa, et al., “Deep-UV tipenhanced Raman scattering," J. Raman Spectrosc., vol. 40, pp. 1324-1330, 2009.

[141] S. K. Jha, Z. Ahmed, M. Agio, et al., "Deep-UV surface-enhanced resonance Raman scattering of adenine on aluminum nanoparticle arrays," J. Am. Chem. Soc., vol. 134, pp. 1966-1969, 2012.

[142] A. Arbabi, E. Arbabi, S. M. Kamali, et al., "Miniature optical planar camera based on a wide-angle metasurface doublet corrected for monochromatic aberrations," Nat. Commun., vol. 7, p. 13682, 2016.

[143] S. Colburn, A. Zhan, and A. Majumdar, "Metasurface optics for full-color computational imaging," Sci. Adv., vol. 4, p. eaar2114, 2018.

[144] H. Liang, Q. Lin, X. Xie, et al., "Ultrahigh numerical aperture metalens at visible wavelengths," Nano Lett., vol. 18, pp. 4460-4466, 2018.

[145] K. Huang, H. F. Gao, G. W. Cao, et al., "Design of DPE for modulating the electric field at the out-of-focus plane in a lens system," Appl. Opt., vol. 51, pp. 5149-5153, 2012.

[146] L. Huang, X. Chen, H. Mühlenbernd, et al., "Three-dimensional optical holography using a plasmonic metasurface," Nat. Commun., vol. 4, p. 2808, 2013.

[147] D. Wen, F. Yue, G. Li, et al., "Helicity multiplexed broadband metasurface holograms," Nat. Commun., vol. 6, p. 8241, 2015.

[148] K. Huang, "Chirality and antiferromagnetism in optical metasurfaces," in Chirality, Magnetism and Magnetoelectricity, E. Kamenetskii, Ed., Cham, Switzerland, Springer Nature Switzerland AG, 2021.

[149] A. Nemati, Q. Wang, M. Hong, et al., "Tunable and reconfigurable metasurfaces and metadevices," Opto-Electron. Adv., vol. 1, p. 180009, 2018.

[150] X. Zhou, S. He, G. Liu, et al., "New developments in laserbased photoemission spectroscopy and its scientific applications: a key issues review," Rep. Prog. Phys., vol. 81, p. 062101, 2018.

[151] G. Liu, G. Wang, Y. Zhu, et al., "Development of a vacuum ultraviolet laser-based angle-resolved photoemission system with a superhigh energy resolution better than $1 \mathrm{meV}$," Rev. Sci. Instrum., vol. 79, p. 023105, 2008.

[152] H. Iwasawa, E. F. Schwier, M. Arita, et al., "Development of laser-based scanning $\mu$-ARPES system with ultimate energy and momentum resolutions," Ultramicroscopy, vol. 182, pp. 85-91, 2017. 
[153] I. Cucchi, I. Gutiérrez-Lezama, E. Cappelli, et al., "Microfocus laserangle-resolved photoemission on encapsulated mono-, bi-, and few-layer 1T'-WTe ${ }_{2}$," Nano Lett., vol. 19, pp. 554-560, 2018.

[154] F. Qin, K. Huang, J. Wu, et al., "Shaping a subwavelength needle with ultra-long focal length by focusing azimuthally polarized light," Sci. Rep., vol. 5, p. 09977, 2015.

[155] Z. Wang, G. Yuan, M. Yang, et al., "Exciton-enabled meta-optics in two-dimensional transition metal dichalcogenides," Nano Lett., vol. 20, pp. 7964-7972, 2020.

[156] G. H. Yuan, E. T. Rogers, and N. I. Zheludev, "Achromatic superoscillatory lenses with sub-wavelength focusing," Light Sci. Appl., vol. 6, p. e17036, 2017.

[157] H. Liu, M. Q. Mehmood, K. Huang, et al., "Twisted focusing of optical vortices with broadband flat spiral zone plates," Adv. Opt. Mater., vol. 2, pp. 1193-1198, 2014.

[158] K. Huang, H. Liu, G. Si, et al., "Photon-nanosieve for ultrabroadband and large-angle-of-view holograms," Laser Photonics Rev., vol. 11, p. 1700025, 2017.

[159] K. Huang, H. Liu, F. J. Garcia-Vidal, et al., "Ultrahigh-capacity non-periodic photon sieves operating in visible light," Nat. Commun., vol. 6, p. 7059, 2015.

[160] S. Mei, M. Q. Mehmood, S. Hussain, et al., "Flat helical nanosieves," Adv. Funct. Mater., vol. 26, pp. 5255-5262, 2016.

[161] Y. J. Liu, H. Liu, E. S. P. Leong, et al., "Fractal holey metal microlenses with significantly suppressed side lobes and highorder diffractions in focusing," Adv. Opt. Mater., vol. 2, pp. 487-492, 2014.

[162] L. Kipp, M. Skibowski, R. L. Johnson, et al., "Sharper images by focusing soft X-rays with photon sieves," Nature, vol. 414, pp. 184-188, 2001.

[163] C. W. Hsu, B. Zhen, A. D. Stone, et al., "Bound states in the continuum," Nat. Rev. Mater., vol. 1, p. 16048, 2016.

[164] C. Huang, C. Zhang, S. Xiao, et al., "Ultrafast control of vortex microlasers," Science, vol. 367, pp. 1018-1021, 2020.

[165] J. Hu, M. Lawrence, and J. A. Dionne, "High quality factor dielectric metasurfaces for ultraviolet circular Dichroism spectroscopy," ACS Photonics, vol. 7, pp. 36-42, 2020.

[166] V. R. Manfrinato, J. Wen, L. Zhang, et al., "Determining the resolution limits of electron-beam lithography: direct measurement of the point-spread function," Nano Lett., vol. 14, pp. 4406-4412, 2014.
[167] Y. Deng, Q. Huang, Y. Zhao, et al., “Precise fabrication of a $5 \mathrm{~nm}$ graphene nanopore with a helium ion microscope for biomolecule detection," Nanotechnology, vol. 28, p. 045302 , 2016.

[168] T. H. Erika, D. J. April, A. S. Charles, et al., “Charge-coupled devices detectors with high quantum efficiency at UV wavelengths," J. Astron. Telesc. Instrum. Syst., vol. 2, pp. 1-11, 2016.

[169] T. Masuzawa, M. Onishi, I. Saito, et al., "High quantum efficiency UV detection using a-Se based photodetector," Phys. Status Solidi Rapid Res. Lett., vol. 7, pp. 473-476, 2013.

[170] T. Masuzawa, S. Kuniyoshi, M. Onishi, et al., "Conditions for a carrier multiplication in amorphous-selenium based photodetector," Appl. Phys. Lett., vol. 102, p. 073506, 2013.

[171] J. Bai, X. Zhong, S. Jiang, et al., “Graphene nanomesh," Nat. Nanotechnol., vol. 5, pp. 190-194, 2010.

[172] M. Corso, W. Auwärter, M. Muntwiler, et al., “Boron nitride nanomesh,” Science, vol. 303, pp. 217-220, 2004.

[173] C. W. Hsu, B. Zhen, A. D. Stone, et al., "Bound states in the continuum,” Nat. Rev. Mater., vol. 1, pp. 1-13, 2016.

[174] P. Wang, Y. Zheng, X. Chen, et al., "Localization and delocalization of light in photonic moiré lattices," Nature, vol. 577, pp. 42-46, 2020.

[175] Z. Xu and B. M. Sadler, "Ultraviolet communications: potential and state-of-the-art," IEEE Commun. Mag., vol. 46, pp. 67-73, 2008.

[176] X. Yang and D. Wang, "Photocatalysis: from fundamental principles to materials and applications," ACS Appl. Energy Mater., vol. 1, pp. 6657-6693, 2018.

[177] C. Wang, W.-C. D. Yang, D. Raciti, et al., "Endothermic reaction at room temperature enabled by deep-ultraviolet plasmons," Nat. Mater., vol. 20, pp. 346-352, 2020.

[178] K. Deisseroth, “Optogenetics,” Nat. Methods, vol. 8, pp. 26-29, 2011.

[179] K. Zoschke, N. Dietrich, H. Börnick, et al., “UV-based advanced oxidation processes for the treatment of odour compounds: efficiency and by-product formation," Water Res., vol. 46, pp. 5365-5373, 2012.

[180] S. A. Asher, "UV resonance Raman spectroscopy for analytical, physical, and biophysical chemistry. Part 1," Anal. Chem., vol. 65, pp. 59A-66A, 1993. 